\title{
Characterization and functional analysis of phytoene synthase gene family in tobacco
}

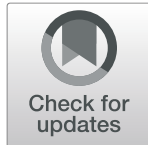

Zhaojun Wang ${ }^{1 \dagger}$, Lin Zhang ${ }^{1,2,3 \dagger}$, Chen Dong ${ }^{2}$, Jinggong Guo ${ }^{4}$, Lifeng Jin $^{2}$, Pan Wei ${ }^{2}$, Feng Li Xiaoquan Zhang ${ }^{1 *}$ and Ran Wang ${ }^{2,5^{*}}$

\begin{abstract}
Background: Carotenoids play important roles in photosynthesis, hormone signaling, and secondary metabolism. Phytoene synthase (PSY) catalyzes the first step of the carotenoid biosynthetic pathway. In this study, we aimed to characterize the PSY genes in tobacco and analyze their function.

Results: In this study, we identified three groups of PSY genes, namely PSY1, PSY2, and PSY3, in four Nicotiana species; phylogenetic analysis indicated that these genes shared a high similarity with those in tomato but not with those in monocots such as rice and maize. The expression levels of PSY1 and PSY2 were observed to be highest in leaves compared to other tissues, and they could be elevated by treatment with certain phytohormones and exposure to strong light. No PSY3 expression was detected under these conditions. We constructed virus-induced PSY1 and PSY2 silencing in tobacco and found that the newly emerged leaves in these plants were characterized by severe bleaching and markedly decreased carotenoid and chlorophyll content. Thylakoid membrane protein complex levels in the gene-silenced plants were also less than those in the control plants. The chlorophyll fluorescence parameters such as Fv/Fm, DPSII, qP, and NPQ, which reflect photosynthetic system activities, of the gene-silenced plants were also significantly decreased. We further performed RNA-Seq and metabonomics analysis between gene-silenced tobacco and control plants. RNA-Seq results showed that abiotic stress, isoprenoid compounds, and amino acid catabolic processes were upregulated, whereas the biosynthesis of cell wall components was downregulated. Metabolic analysis results were consistent with the RNA-Seq. We also found the downstream genes in carotenoid biosynthesis pathways were upregulated, and putative transcription factors that regulate carotenoid biosynthesis were identified.
\end{abstract}

Conclusions: Our results suggest that PSY can regulate carotenoid contents not only by controlling the first biosynthesis step but also by exerting effects on the expression of downstream genes, which would thereby affect photosynthetic activity. Meanwhile, PSY may affect other processes such as amino acid catabolism and cell wall organization. The information we report here may aid further research on PSY genes and carotenoid biosynthesis.

Keywords: Carotenoids, Phytoene synthase, Tobacco

\footnotetext{
* Correspondence: zhangxquan@henau.edu.cn; wangr@ztri.com.cn

†Zhaojun Wang and Lin Zhang contributed equally to this work.

'College of Tobacco Science, Henan Agricultural University, Zhengzhou 450002, China

${ }^{2}$ China Tobacco Gene Research Center, Zhengzhou Tobacco Research Institute, Zhengzhou 450001, China

Full list of author information is available at the end of the article
}

(c) The Author(s). 2021 Open Access This article is licensed under a Creative Commons Attribution 4.0 International License, which permits use, sharing, adaptation, distribution and reproduction in any medium or format, as long as you give appropriate credit to the original author(s) and the source, provide a link to the Creative Commons licence, and indicate if changes were made. The images or other third party material in this article are included in the article's Creative Commons licence, unless indicated otherwise in a credit line to the material. If material is not included in the article's Creative Commons licence and your intended use is not permitted by statutory regulation or exceeds the permitted use, you will need to obtain permission directly from the copyright holder. To view a copy of this licence, visit http://creativecommons.org/licenses/by/4.0/ The Creative Commons Public Domain Dedication waiver (http://creativecommons.org/publicdomain/zero/1.0/) applies to the data made available in this article, unless otherwise stated in a credit line to the data. 


\section{Background}

Carotenoids are widely found in photosynthetic organisms, including plants, algae, and cyanobacteria. Chemically, carotenoids belong to isoprenoid compounds; typical carotenoids contain 40 carbon atoms (C40) that are formed by the condensation of eight $\mathrm{C} 5$ isoprenoid units. The number of conjugated double bounds in their chemical structure confers them a visible-light absorption property that produces their characteristic color of yellow to red $[1,2]$. Carotenoids contain a large number of different components; at present, nearly 1200 natural carotenoids have been found in 700 organisms from all domains of life. Carotenoids that do not contain oxygen are classified as carotenes, and those that contain oxygen are classified as xanthophylls. In addition to the typical C40 carotenoids, some carotenoids that are shorter (C30) or longer (C45 or C50) have also been found [3].

Although humans do not metabolically synthesize carotenoids, carotenoids can be acquired via the consumption of food or supplementation. As naturally occurring pigments, carotenoids have a range of functions in human health. Carotenoids are important antioxidants as they absorb specific wavelengths of light and are the precursors of vitamin A. Moreover, they play important roles in protecting the eyes and in maintaining normal vision. Furthermore, they may protect against certain types of cancer by enhancing cell communication, suppressing abnormal cell growth, or providing UV protection. Carotenoids can prevent heart disease by reducing oxidized low-density lipoproteins $[1,4]$.

Carotenoids are also indispensable in plants. They provide protection against photooxidative damage; photoprotection is one of their most important functions. Under strong light conditions, carotenoids can dissipate excess energy as heat, eliminate free radicals, and prevent the lipid peroxidation of membranes, thereby enhancing the adaptation of plants to different light conditions [5]. Another important function of carotenoids is that it has a role in the reaction center of photosystem II. Carotenoids promote the formation of pigment-protein complexes and assist in energy absorption and electron flow transport [6]. In plants, carotenoids can serve as precursors to phytohormones such as abscisic acid (ABA) [7] and strigolactones [8], which both play vital roles in plant development and stress responses. Additionally, carotenoids play important roles in plant reproduction: the different colors that they give can attract animals that help in pollination and seed dispersal [9].

The biosynthesis of carotenoids in plants is part of the isoprenoid precursor metabolism. Starting from isopentenyl diphosphate (IPP) and dimethylallyl diphosphate (DMAPP), the biosynthesis of carotenoids is catalyzed by a series of enzymes [3]; the first step is the generation of geranylgeranyl diphosphate (GGPP) through the addition of three IPP molecules to one DMAPP, whose conversion is catalyzed by GGPP synthetase (GGPPS). GGPP is a precursor to several groups of other isoprenoids [10]. The next step in carotenoid biosynthesis is the production of 40-carbon phytoene through the condensation of two GGPP molecules; this condensation reaction is catalyzed by the enzyme phytoene synthase (PSY) and is considered the main "bottleneck" in the carotenoid biosynthetic pathway [11]. Then, phytoene is then converted to lycopene through a series of desaturation and isomerization reactions. Two types of phytoene desaturases, namely phytoene desaturase (PDS) [12] and $\zeta$-carotene desaturase (ZDS) [13], are reportedly responsible for the desaturation reactions, whereas 15 cis- $\zeta$-carotene isomerase (Z-ISO) catalyzes the isomerization reactions [14]. The next step is the cyclization of lycopene, wherein two branches, namely $\alpha$ - and $\beta$ branches, which both are converted into different components, are formed. The $\alpha$-branch is relatively simple; lycopene is cyclized into $\delta$-carotene with the help of lycopene $\varepsilon$-cyclase (LCYE) [15] and further cyclized into $\alpha$-carotene by lycopene $\beta$-cyclase (LCYB) [16]. $\alpha$ carotene can be hydroxylated by two types of carotenoid hydroxylases. Carotenoid $\beta$-hydroxylase (CHYB, mainly cytochrome $\mathrm{P} 450$ enzymes, CYP97 type) produce zeinoxanthin which is further hydroxylated by carotenoid $\varepsilon$-hydroxylase (CHYE, mainly CYP97C1) into lutein. Compared with the $\alpha$-branch, the $\beta$-branch contains relatively more steps that lead to many intermediate products. First, lycopene undergoes two rounds of cyclization that is catalyzed by LCYB and leads to the production of $\gamma$ - and $\beta$-carotene. Then, $\beta$-carotene undergoes two steps of hydroxylation reaction that is catalyzed by $\beta$-carotene hydroxylase $(\mathrm{BCH})$ and leads to the production of $\beta$-cryptoxanthin and zeaxanthin [17]. Next, a two-step cyclization reaction of zeaxanthin is catalyzed by the enzyme zeaxanthin epoxidase (ZEP) and forms antheraxanthin and violaxanthin. The last step in the $\beta$-branch of the carotenoid biosynthetic pathway is the conversion of violaxanthin into neoxanthin; this conversion is catalyzed by neoxanthin synthase (NXS) [11].

PSY catalyzes the biosynthesis of phytoene from GGPP, which is a common precursor of many other isoprenoids [10]. The formation of phytoene is the first step in carotenoid biosynthesis and the main bottleneck step [18]. PSYs are encoded by small gene families; the genes encoding PSY have been identified and isolated in many species such as Arabidopsis [19], rice [20], maize [21], and tomato [22-24], and their function and expression patterns have been previously reported [25]. In Arabidopsis, the PSY gene is expressed in not only photosynthetic tissues but also non-photosynthetic tissues, 
including roots, in trace amounts and has a pattern of co-expression with other carotenoid pathway genes [26], indicating that the PSY gene is involved mainly in photosynthetic pathways. The expression of $P S Y$ genes is regulated by various factors, including developmental and environmental signals [27]. Phytohormones, especially ethylene, play an important role in the regulation of $P S Y$ gene expression; increased ethylene levels significantly upregulate the transcription of PSY genes [28]. Abscisic acid can also regulate the expression of PSY genes [25]. Environmental signals such as strong light, salt, drought, temperature, and photoperiod can also modify the expression levels of PSY genes [29]. Some important transcription factors were found to perceive the signals mentioned above and in turn control the transcription of PSY genes; for example, PHYTOCHROME INTERA CTING FACTOR 1 (PIF1) [30] and LONG HYPOCOTYL 5 (HY5) [31], which belong to bHLH and bZIP families, respectively, were proven to be involved in the light-induced regulation of PSY gene expression. At the protein level, PSYs are also regulated; the regulation of PSYs include the localization of PSY within the chloroplast; this localization influence their bioavailability [32]. Furthermore, carotenoid metabolites have been found to negatively regulate PSY protein levels [33].

Similar to other plants, carotenoids also play an important role in photosynthesis, physiological processes, and stress responses in tobacco [34]. In addition, due to the properties of tobacco having huge biomass and being easy to genetically modified, tobacco is considered an ideal species from which to obtain valuable carotenoid components [35]. PSYs control the metabolic flux of carotenoids, making the functions of tobacco PSYs notable for studying. In a previous study, two transcripts were cloned from Nicotiana tabacum cultivar Petit Havana SR1 and showed $86 \%$ identity in both nucleotide and amino acid sequences [36]. The overexpression of both genes resulted in a severe dwarf phenotype, changes in pigment composition, and high levels of phytoene; these confirm the importance of the role of PSYs in controlling tobacco carotenoid biosynthesis. However, the two sequences were obtained by using homology-based cloning. The reference genome sequences of some Nicotiana species, such as $N$. tabacum [37, 38], $N$. benthamiana [39], N. sylvestris, and N. tomentosiformis [40] have been released. Thus, the aim of this study was to survey PSY coding genes at the genome level and extensively study their functions in carotenoid biosynthesis and photosynthesis such that more information about this gene family is obtained.

\section{Results}

\section{Identification of PSY genes in tobacco}

BLAST analysis was performed by querying Arabidopsis PSY protein sequences from different tobacco genomes, and 6, 5, 3, and 3 candidate $P S Y$ genes were found in $N$.

Table 1 PSY genes identified in four Nicotiana species

\begin{tabular}{|c|c|c|c|c|c|c|c|c|c|c|}
\hline & \multirow[t]{2}{*}{ Gene name } & \multirow[t]{2}{*}{ Gene ID } & \multirow{2}{*}{$\begin{array}{l}\text { Exon } \\
\text { number }\end{array}$} & \multirow{2}{*}{$\begin{array}{l}\text { MW } \\
\text { (KDa) }\end{array}$} & \multirow[t]{2}{*}{ PI } & \multirow{2}{*}{$\begin{array}{l}\text { CDS } \\
\text { (bp) }\end{array}$} & \multirow{2}{*}{$\begin{array}{l}\text { Length } \\
\text { (aa) }\end{array}$} & \multicolumn{3}{|c|}{ Pfam Matches } \\
\hline & & & & & & & & ID & Start & End \\
\hline \multirow[t]{6}{*}{ N. tabacum } & NtPSY1-1 & mRNA_24760_cds & 7 & 46.53 & 7.53 & 1233 & 410 & PF00494 & 129 & 384 \\
\hline & NtPSY1-2 & mRNA_28821_cds & 7 & 46.56 & 8.1 & 1233 & 410 & PF00494 & 129 & 384 \\
\hline & NtPSY2-1 & mRNA_108630_cds & 7 & 49.55 & 8.98 & 1323 & 440 & PF00494 & 155 & 410 \\
\hline & $N t P S Y 2-2$ & mRNA_3350_cds & 7 & 49.72 & 9.16 & 1326 & 441 & PF00494 & 156 & 411 \\
\hline & NtPSY3-1 & mRNA_22099_cds & 6 & 43.75 & 8.71 & 1146 & 381 & PF00494 & 104 & 358 \\
\hline & NtPSY3-2 & mRNA_111132_cds & 6 & 43.83 & 8.51 & 1146 & 381 & PF00494 & 103 & 358 \\
\hline \multirow[t]{5}{*}{ N. benthamiana } & NibenPSY1-1 & Niben101Scf01959g00004 & 8 & 46.52 & 6.78 & 1233 & 410 & PF00494 & 129 & 384 \\
\hline & NibenPSY1-2 & Niben101Scf04020g00002 & 8 & 50.29 & 7.51 & 1326 & 441 & PF00494 & 129 & 382 \\
\hline & NibenPSY2 & Niben101Scf07253g01008 & 8 & 49.64 & 8.75 & 1323 & 440 & PF00494 & 157 & 412 \\
\hline & NibenPSY3-1 & Niben101Scf08679g04027 & 6 & 44.14 & 8.60 & 1146 & 381 & PF00494 & 103 & 358 \\
\hline & NibenPSY3-2 & Niben101Scf04118g01004 & 7 & 34.86 & 6.74 & 924 & 307 & PF00494 & 83 & 252 \\
\hline \multirow[t]{3}{*}{ N. sylvestris } & NsylPSY1 & mRNA_81209_cds & 7 & 46.53 & 7.53 & 1233 & 410 & PF00494 & 129 & 384 \\
\hline & NsyIPSY2 & mRNA_73510_cds & 7 & 49.34 & 8.98 & 1317 & 438 & PF00494 & 155 & 410 \\
\hline & NsylPSY3 & mRNA_53352_cds & 6 & 43.90 & 8.63 & 1146 & 381 & PF00494 & 103 & 358 \\
\hline \multirow[t]{3}{*}{ N. tomentosiformis } & NtomPSY1 & mRNA_60982_cds & 7 & 46.56 & 8.10 & 1314 & 410 & PF00494 & 129 & 384 \\
\hline & NtomPSY2 & mRNA_59648_cds & 7 & 49.51 & 9.16 & 1320 & 439 & PF00494 & 156 & 411 \\
\hline & NtomPSY3 & mRNA_83828_cds & 6 & 43.83 & 8.51 & 1146 & 381 & PF00494 & 103 & 358 \\
\hline
\end{tabular}

The gene IDs shown were extracted from the genomic annotation information of each species deposited in Sol Genomics Network (SGN) database (https:// solgenomics.net/). The genome version of N. tabacum used here was reported by Sierro et al., 2014 [37] 
tabacum, N. benthamiana, N. sylvestris, and N. tomentosiformis, respectively. Their temporary names and molecular characteristics are shown in Table 1. The coding sequence length of tobacco PSYs ranged from 924 to $1326 \mathrm{bp}$, and the resulting protein molecular weights ranged from 34.86 to $50.29 \mathrm{kD}$. The isoelectric point of PSYs ranged from 6.74 to $9.16 \mathrm{pH}$, indicating that these proteins are alkalescent. The exon number of PSYS ranged from six to eight (Table 1).

\section{Phylogenetic analysis of tobacco PSY gene family}

The phylogenetic relationships of tobacco PSY genes and homologs in Arabidopsis, rice, maize, and tomato were analyzed using MEGA 5 software. PSY genes from different tobacco species can be divided into three groups (A, B, and $\mathrm{C}$ ) based on their phylogenetic relationships (Fig. 1). Among them, NtPSY1-1, NtPSY1-2, NibenPSY1-1, NibenPSY1-2, NsylPSY1, and NtomPSY1 were classified under group A, NtPSY2-1, NtPSY2-2, NibenPSY2, NsylPSY2, and NtomPSY2 under group B, and NtPSY3-1, NtPSY3-2, NibenPSY3-1, NibenPSY3-2, NsylPSY3, and NtomPSY3 under group C. In each group, strong correlations among the genes from N. tabacum, $N$. sylvestris, and $N$. tomentosiformis, compared with that of $N$. benthamiana, were observed. Under group A and B, 7 exons and 6 introns were identified in the PSY genes of $N$. tabacum, $N$. sylvestris, and $N$. tomentosiformis, whereas 8 exons and 7 introns were identified in

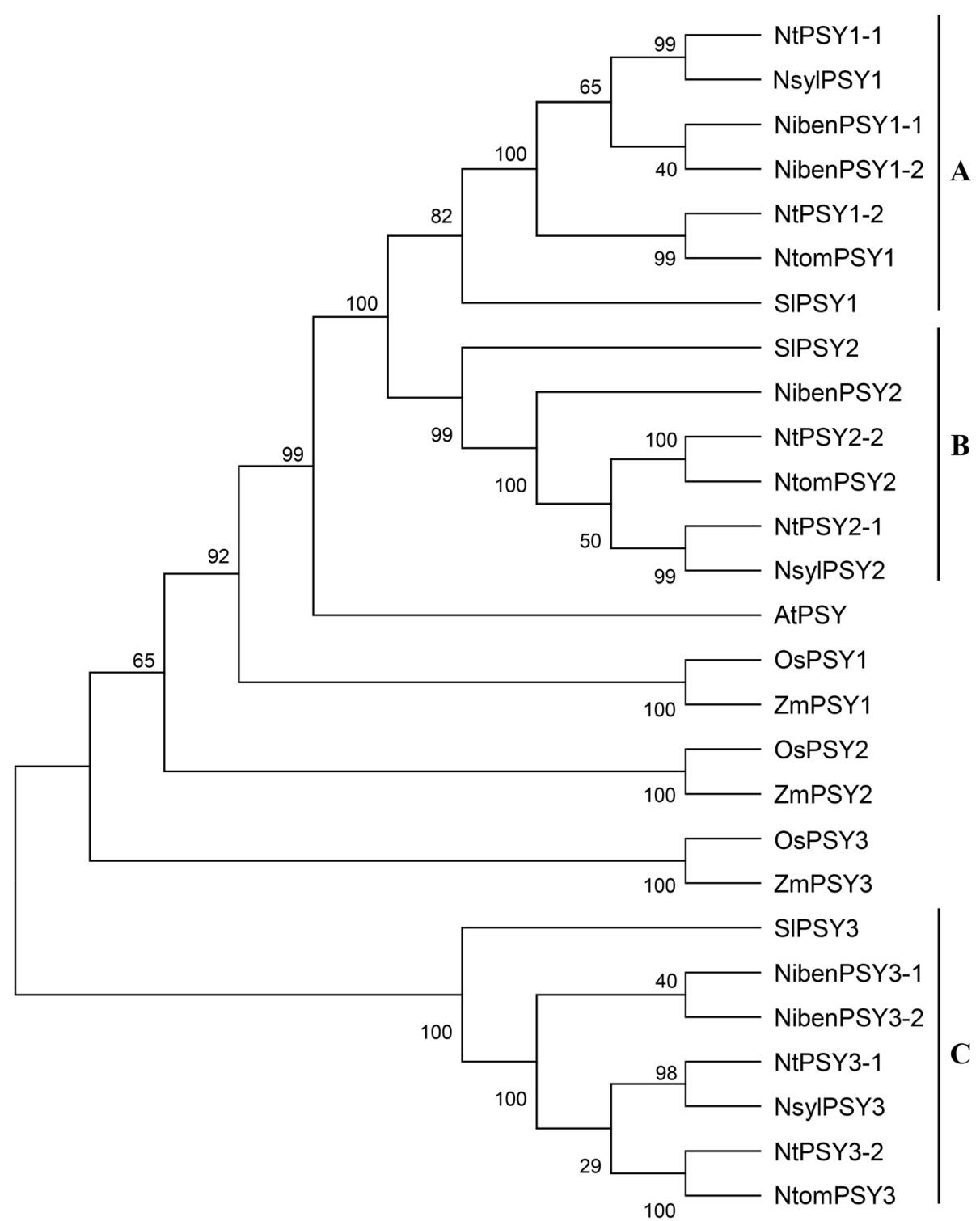

Fig. 1 Phylogenetic analysis of tobacco PSY protein sequences. PSY protein sequences of Arabidopsis, rice, maize, and tomato using sequence accession numbers from a previous study [24] were downloaded from GeneBank database 
the genes of N. benthamiana; for group C, 6 exons and 5 introns were identified in all the genes, except NibenPSY3-2, which contained 7 exons and 6 introns (Table 1). These results indicate a relatively low phylogenetic relationship between $N$. benthamiana and other Nicotiana species.

Three PSY genes in tomato were also clustered with tobacco PSY genes into three groups (Fig. 1), indicating that $P S Y$ gene sequences are conserved in Solanaceae species. However, PSYs in Arabidopsis, rice, and maize were not clustered with those in tobacco and tomato, suggesting that the sequences of PSYs among these species and Solanaceae were diverse.

\section{Cis-element analysis of NtPSY promoters}

We used $N$. tabacum as a model to survey cis-elements in tobacco PSY gene promoters. Fragments of $2000 \mathrm{bp}$ upstream of the start codons of 6 NtPSY genes were extracted from tobacco genomic sequences and queried against PlantCARE database (http://bioinformatics.psb. ugent.be/webtools/plantcare/html/). As shown in Fig. 2, most of the cis-elements found were involved in responses to light (ACE, AE-box, ATCT-motif, Box 4, Box II, chs-CMA1a, chs-CMA2a, GA-motif, GATA-motif, GATT-motif, G-Box, LAMP-element, GT1-motif, MRE, and TCT-motif). Other cis-elements were identified to be involved in responses to temperature (LTR), drought stress (MYB, TC-rich), or phytohormones, including MeJA (CGTCA-motif, TGACG-motif), abscisic acid (ABRE), auxin (TGA-element), gibberellin (GARE-motif, TATC-box, P-box), and salicylic acid (TCA-element), indicating that the expression of PSYS is regulated by a wide range of developmental and environmental factors.

Among these cis-elements, box 4, GATA-motif, Gbox, TCT-motif, MYC, ABRE, ERE, ARE, and MYB were present in all three groups of NtPSY (Additional file 1: Table S1). AE-box, ATCT-motif, TGACT-motif, CGTCA-motif, and GCN4-motif were present in only NtPSY1. chs-CMA2a, GA-motif, TATC-box, P-box, TCA-element, and $\mathrm{W}$ box were present exclusively in
NtPSY2. chs-CMA1a, GATT-motif, LAMP-element, and TGA-element were solely present in NtPSY3. The diversity of cis-elements in different NtPSY promoters indicates that their expression may be regulated by different mechanisms.

\section{Expression pattern of NtPSY genes in tissues}

The gene expression levels of NtPSY in four tissues (leaf, stem, flower, and root) at full-bloom stage were compared. Due to the high similarity among NtPSY genes, three pairs of conserved qPCR primers (see Additional file 2: Table S2) that can be used to estimate the sum expression of NtPSY1-1 and NtPSY1-2, NtPSY2-1 and NtPSY2-2, and NtPSY3-1 and NtPSY3-2, respectively, were designed. The results indicated that the expression of NtPSY3-1 and NtPSY3-2 was not detectable in any of the four tissues (data not shown), indicating that they possibly are not expressed in these tissues. Similar expression patterns were identified in the other four genes; the highest expression levels were found in leaves, intermediate levels in stems and flowers, and relatively low levels in roots (Fig. 3a), indicating that $N t P S Y$ genes function mainly in leaves, stems, and flowers. In addition, the expression levels of NtPSY1-1 and NtPSY1-2 were much higher than those of $N t P S Y 2-1$ and NtPSY2-2, suggesting that NtPSY1-1 and NtPSY1-2 are functionally more important than NtPSY2-1 and NtPSY2-2.

\section{The expression of NtPSY genes are influenced by different phytohormones and strong light conditions}

To obtain the expression profiles of tobacco PSY genes under phytohormone treatment and strong light conditions, $N$. tabacum was used as a model, treated with abscisic acid (ABA), methyl jasmonate (MeJA), indole-3acetic acid (IAA), 6-benzyladenine (6-BA), gibberellin (GA), and exposed to strong light. qPCR was performed to determine the relative expression levels of NtPSY genes under different treatments. No expression for NtPSY3-1 and NtPSY3-2 was detected after any of the

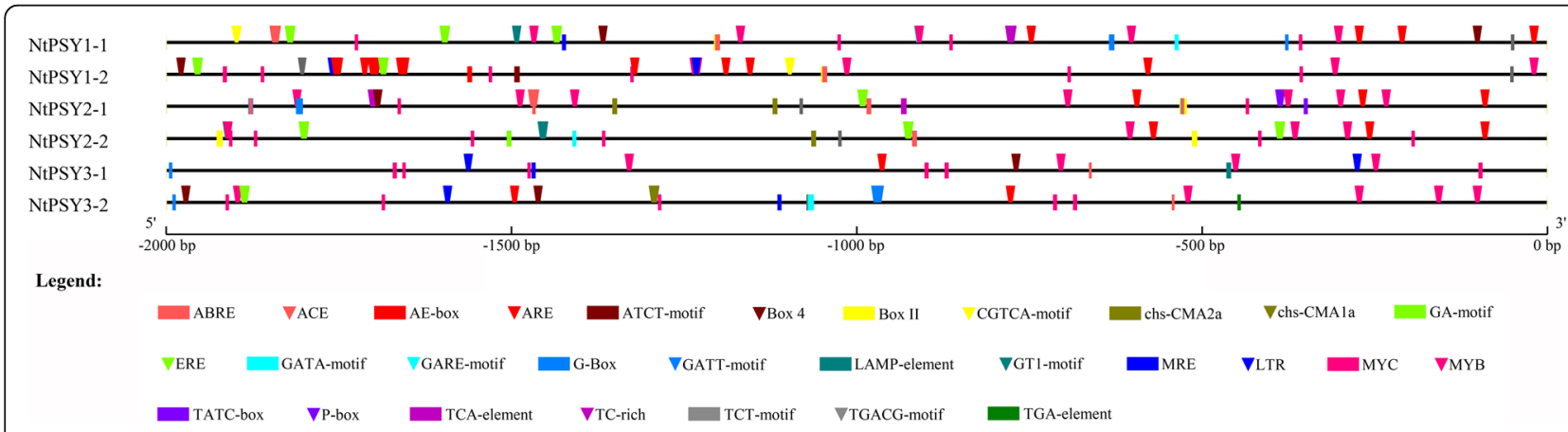

Fig. 2 Cis-elements of NtPSY gene promoter. A fragment of 2000 bp upstream of the start codons of each NtPSY gene were analyzed. The core promoter elements such as TATA-box and CAAT-box were masked for clarity 

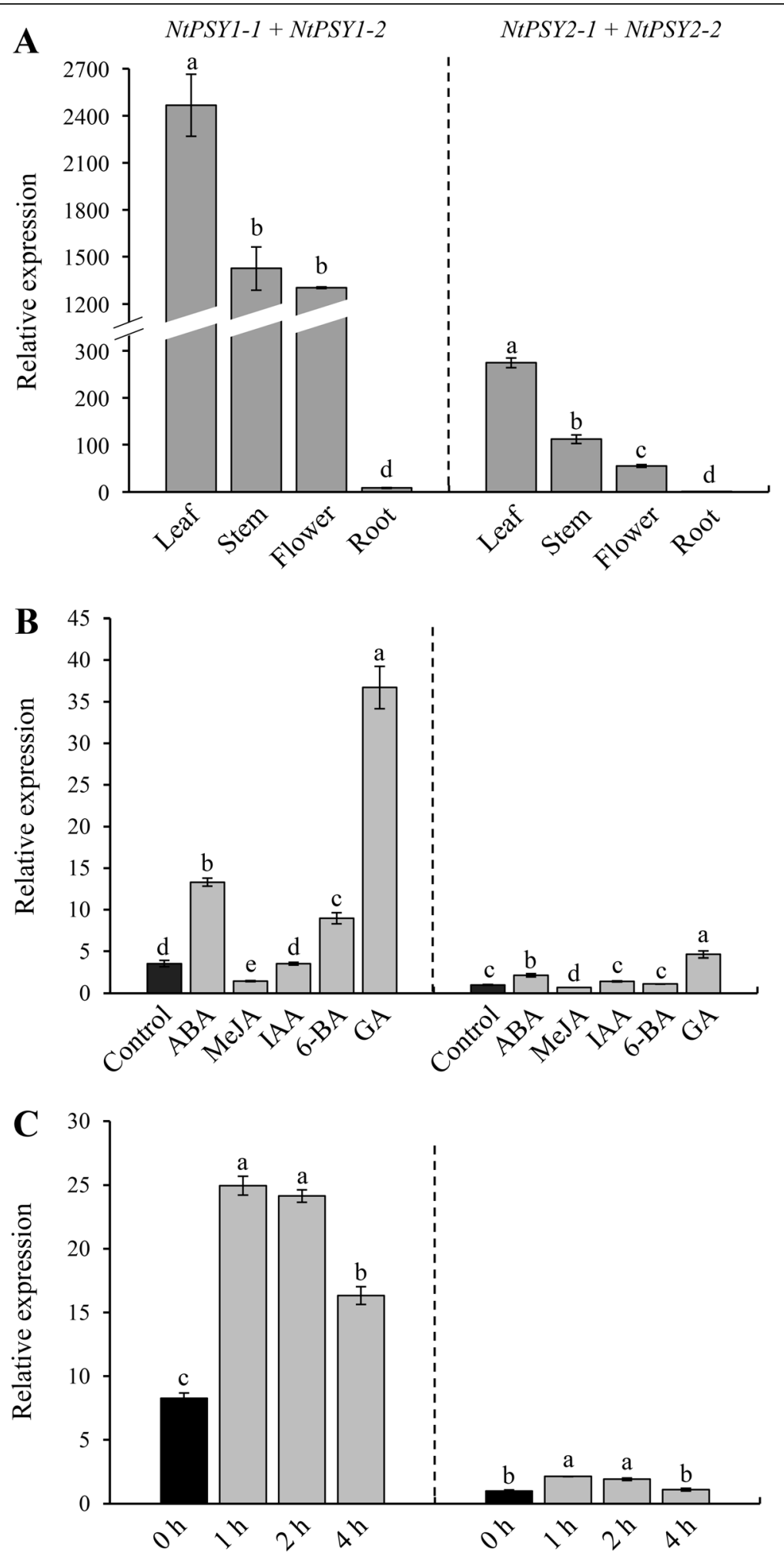

Fig. 3 (See legend on next page.) 
(See figure on previous page.)

Fig. 3 Expression pattern of NtPSY genes. a Expression levels of NtPSY genes in leaves, stems, flowers, and roots; the relative expression levels in each tissue were calculated by setting the expression value of NtPSY2-1 + NtPSY2-2 in roots as 1. $\mathbf{b}$ Changes in the expression of NtPSY genes under treatment with different phytohormones; the relative expression levels under different conditions were calculated by setting the expression value of NtPSY2-1 + NtPSY2-2 in the control as 1. c Changes in the expression of NtPSY genes under strong light conditions; the relative expression levels at different intervals were calculated by setting the expression value of NtPSY2-1 + NtPSY2-2 at $0 \mathrm{~h}$ as 1 . Three pairs of conserved qPCR primers that can be used to estimate the sum expression of NtPSY1-1 and NtPSY1-2, NtPSY2-1 and NtPSY2-2, NtPSY3-1 and NtPSY3-2, respectively, were designed. Columns and bars represent the means and standard errors $(n=3)$, respectively. Columns marked by different letters indicate statistical significance $(P<0.05)$. The data for the expression of NtPSY3-1 and NtPSY3-2 are not shown as no expression was detected under all the conditions

treatments (data not shown). After the treatment of $N$. tabacum with ABA, 6-BA, and GA, the expression levels of the other four genes were significantly upregulated, but significantly downregulated in the $N$. tabacum treated with MeJA; no marked changes in expression levels were identified after IAA treatment (Fig. 3b). Under strong light conditions, the expression of NtPSY1-1, NtPSY1-2, NtPSY2-1, and NtPSY2-2 were all upregulated and reached a peak after $1 \mathrm{~h}$ of treatment and declined thereafter (Fig. 3c). The expression levels of NtPSY1-1 and NtPSY1-2 were markedly higher than those of NtPSY2-1 and NtPSY2-2 under all treatments. These results indicate that phytohormones and light play important roles in regulating NtPSY gene expression.

\section{Virus-induced NbibenPSY gene silencing}

To further investigate the function of tobacco PSY genes, we generated two virus-induced gene silencing constructs, namely TRV-PSY1 and TRV-PSY2. The former contains a conserved fragment shared by NibenPSY1-1 and NibenPSY1-2 and can silence both of them, whereas the latter can silence NibenPSY2. The two constructs were co-introduced into $N$. benthamiana by agrobacterium-mediated transformation to silence these three genes simultaneously; distilled water, empty vector, and TRV-PDS construct (can silence the phytoene desaturase gene as previously described [41]) were defined as blank, negative, and positive control, respectively. As shown in Fig. 4a-d, the newly emerged leaves of the positive control and TRV-PSY1/TRV-PSY2 cotransformed plants (named TRV-PSY1\&2 hereafter) were severely bleached and characterized by abnormally wrinkled shapes, whereas no marked changes were identified in the blank and negative controls. Conserved qPCR primers were designed to estimate the expression levels of all three NibenPSY genes. The expression levels of NibenPSY genes was markedly suppressed in TRVPSY1\&2 plants compared to those of the blank and negative controls (Fig. 4e). These results suggest that the NibenPSY genes were silenced.

\section{Photosystem changes in NbibenPSY-silenced plants}

Carotenoids have been long proven to play important roles in plant photosynthesis [6], and the bleached phenotype of NbibenPSY-silenced plants we observed inspired us to analyze potential changes in photosystem. First, we measured carotenoid and chlorophyll content (Fig. 5a). Compared with the negative controls, the carotenoid content in TRV-PSY1\&2 plants was significantly decreased, and only $60 \%$ carotenoids were detected. The contents of chlorophyll a and chlorophyll b were also decreased in TRV-PSY1\&2 plants, with decrements of 67.26 and $64.65 \%$, respectively.

Next, we explored the effects of NbibenPSY silencing on thylakoid structures; thylakoid membrane protein complex was analyzed using blue-native polyacrylamide gel-electrophoresis (BN-PAGE) (Fig. 5b). All protein band densities were decreased in TRV-PSY1\&2 plants compared to the negative control, indicating that there is less accumulation of the thylakoid membrane protein complex in TRV-PSY1\&2 plants.

We measured the chlorophyll fluorescence difference between TRV-PSY1\&2 and negative control plants to evaluate their photosynthetic performance. Four parameters, namely $\mathrm{Fv} / \mathrm{Fm}$ (maximum quantum efficiency of PSII photochemistry), ФPSII (sum of the quantum yields of PSII photochemistry), qP (photochemical quenching), and NPQ (non-photochemical quenching), were measured (Fig. 5c); compared with the measurements of these parameters in the negative control, those in TRVPSY1\&2 plants were significantly decreased, indicating that the photosystem activities in NbibenPSY-silenced plants were significantly decreased.

\section{Metabolite analysis of TRV-PSY1\&2 leaves compared with the control}

The metabolic changes in leaves induced by the silencing of $P S Y$ genes were analyzed using GC-MS. The levels of 85 known metabolites were determined (Additional file 3: Table S3). Most of the compounds, including amino acids and organic acids, were downregulated in TRV-PSY1\&2. Only 16 components were upregulated in TRV-PSY1\&2; these components include cell wall components and mainly sugars and their derivatives, including arabinofuranose, levoglucosan, and arabinitol. Interestingly, sedoheptulose was also among the upregulated components. In plants, sedoheptulose exists mainly as monophosphate, plays vital roles during 


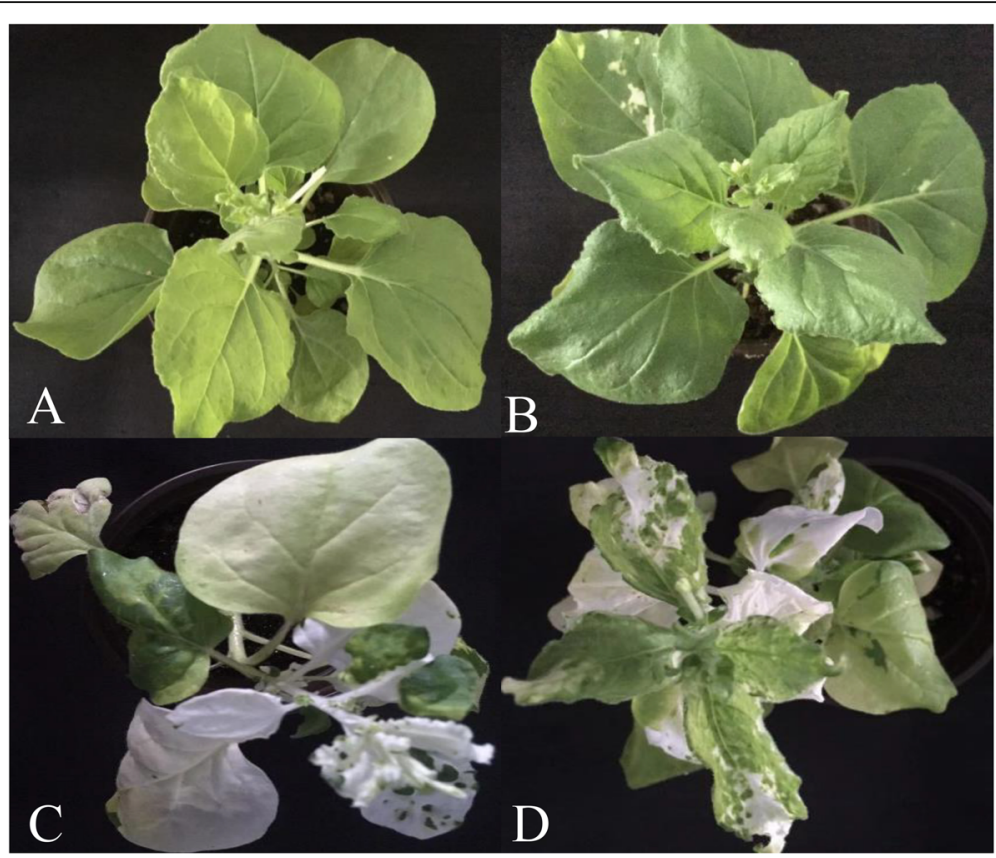

$\mathbf{E}$

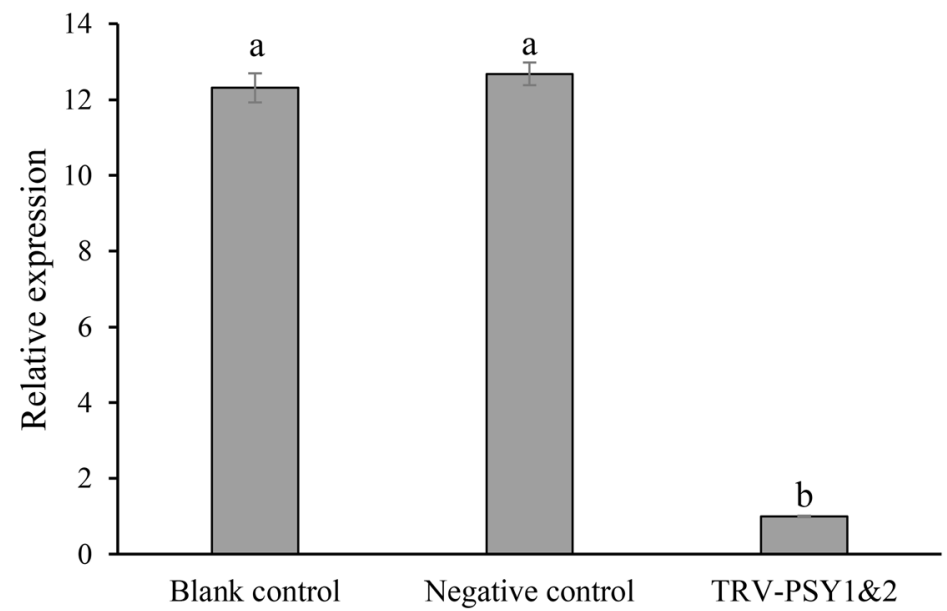

Fig. 4 TRV-mediated PSY gene silencing in N. benthamiana. a Blank control (transformed using distilled water). b Negative control (transformed using empty vector). c Positive control (transformed using TRV-PDS construct). d TRV-PSY1\&2 (co-transformed with TRV-PSY1 and TRV-PSY2). e Total expression of NibenPSY1-1, NibenPSY1-2, and NibenPSY2 in blank control, negative control, and TRV-PSY1\&2 measured by qPCR using conserved primers. The relative expression levels in different plants were calculated by setting the gene expression value in TRV-PSY1\&2 as 1. Columns and bars represent the means and standard erorrs $(n=3)$, respectively. Columns marked by different letters indicate $P<0.05$

photosynthesis, and is liberated only upon cell death [42], indicating that cell death severely occurs in TRVPSY1\&2.

\section{Global analysis of RNA-seq data between TRV-PSY1\&2 and control plants}

To extensively analyze the function of tobacco PSYs, we performed an RNA-Seq analysis between the genes of TRV-PSY1\&2 and negative controls. Three biological replicates were used for each group. Approximately 46 million paired-end raw reads were produced for each sample. Clean reads were obtained by discarding low-quality reads; a total of 270 million clean reads were generated and processed to assemble a de novo transcriptome using Trinity software [43]. A total of 418,816 transcripts were obtained, and each unigene was defined as the longest transcript in a homologous group. Finally, a total of 169, 954 unigenes, with an average contig length of $598 \mathrm{bp}$ and a minimum and maximum length of 201 and 12,283 bp, respectively, were obtained (Table 2). 


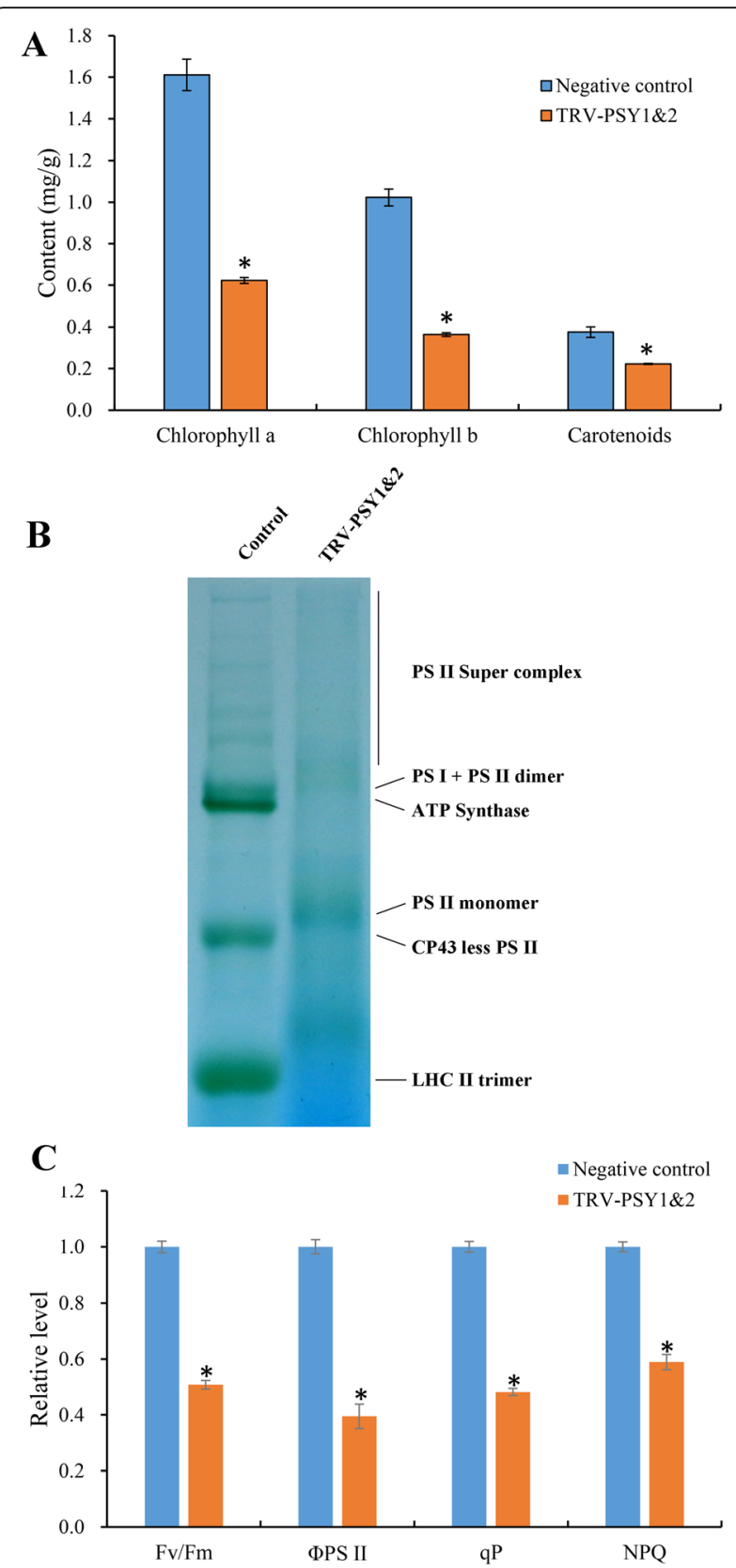

Fig. 5 Photosystem changes in virus-mediated PSY gene silencing in $\mathbf{N}$. benthamiana. a Carotenoid and chlorophyll content. $\mathbf{b}$ Blue-native polyacrylamide gel-electrophoresis analysis of thylakoid membrane protein complex in TRV-PSY1\&2 and negative control plants. c Chlorophyll fluorescence difference between TRVPSY1\&2 and negative control plants; the relative levels of each parameter were calculated by setting the value in the negative control as 1. Columns and bars represent the means and standard errors $(n=9)$, respectively. ${ }^{*}$ indicate $P<0.05$. PS I, photosystem I. PS II, photosystem II. CP 43, 43 kD Chlorophyll a binding protein. LHC II, light harvesting complex II. Fv/Fm, maximum quantum efficiency of PSII photochemistry. DPSII, sum of the quantum yields of PSII photochemistry. qP, photochemical quenching. NPQ, nonphotochemical quenching
The unigenes obtained were queried against and annotated using the following databases: NT (NCBI nucleotide sequences), NR (NCBI non-redundant protein sequences), COG (Clusters of Orthologous Groups of proteins), KOG (euKaryotic Ortholog Groups), SwissProt (A manually annotated and reviewed protein sequence database), TrEMBL, PFAM (Protein family), CDD (Conserved Domain Database), GO (Gene Ontology), and KEGG (Kyoto Encyclopedia of Genes and Genomes). All 169,954 unigenes were annotated; $64.14 \%$ of unigenes were annotated in at least one database and $0.82 \%$ in all databases (Additional file 4: Table S4).

The set of unigenes obtained above was used as a reference sequence, and clean reads of each sample were then mapped to it using Bowtie2 software [44]. For each sample, more than $93 \%$ of the clean reads were successfully mapped (Table 3 ), indicating that the quality of our results was sufficient for downstream analysis.

To facilitate the comparison of differences in gene expression levels between different samples, the gene expression levels for each sample were calculated based on the reads mapping results and are shown as transcripts per million (TPM) values [45].

\section{Functional analysis of differentially expressed genes between TRV-PSY1\&2 and control plants}

Differentially expressed genes (DEGs) were identified using DESeq software [46], with $p$-values and $q$-values < 0.05 and $\log _{2}$ FoldChange $>1$ or $<-1$ as the threshold for significant differential expression. In this study, a total of 748 and 854. DEGs were upregulated and downregulated in TRV-PSY1\&2 plants, respectively (Additional file 5: Table S5). To evaluate the functional categories of these DEGs, GO enrichment analysis was performed using topGO software [47]. A $p<0.05$ and $q$ $<0.05$ were set as the significant threshold, and 58 and 96 GO terms were enriched for these upregulated and downregulated DEGs, respectively (Additional file 6: Table S6). The top 20 biological process GO terms are shown in Fig. 6. The pathways involved in abiotic stress, isoprenoid compounds, and amino acid catabolic processes were upregulated in TRV-PSY1\&2 plants, whereas the downregulated pathways were involved mainly in the biosynthesis of cell wall components, such as polysaccharides, glucans, cellulose, pectin, and galacturonan, indicating that PSY may play an important role in these processes.

\section{Changes in the expression of carotenoid biosynthesis pathway genes}

The changes in the expression levels of NbibenPSY genes in gene-silenced and control plants were examined using the RNA-Seq data, which is consistent with the qPCR analysis (Fig. 4). Their expression was significantly 
Table 2 Length distribution of de novo assembled transcriptome contigs

\begin{tabular}{lllllll}
\hline & Total Nubmer & N50 (bp) & N90 (bp) & Maximum Length (bp) & Minimum Length (bp) & Average Length (bp) \\
\hline Transcript & 418,816 & 1269 & 340 & 12,283 & 201 & 820.65 \\
Unigene & 169,954 & 884 & 250 & 12,283 & 201 & 597.87
\end{tabular}

repressed in TRV-PSY1\&2 plants compared to that of the control plants (Table 4); this further confirms the high quality of the RNA-Seq results. GGPP synthetase, which operates upstream of PSY, was also downregulated in TRV-PSY1\&2; on the contrary, almost all the downstream genes of PSY, except NXS, were upregulated in TRV-PSY1\&2 compared to those of the control plants (Table 4).

\section{qPCR verification of carotenoid biosynthesis genes}

To confirm the RNA-seq results, genes involved in the six steps in carotenoid biosynthesis pathways were randomly selected for qPCR analysis; the genes selected were those encoding GGPPS, PDS, ZDS, CRTISO, $\beta$ LCY, and NXS. For the determination of the total expression levels of genes in each of the selected steps, conserved primers were designed; the primers used are listed in Additional file 2: Table S2. The results indicate that although some quantitative differences at the expression level were present, qRT-PCR results indicated that all of the genes have similar expression patterns as indicated by the RNA-seq data (Additional file 7: Fig. S1), thereby further validating the RNA-Seq data.

\section{Identification of putative transcription factors that regulate carotenoid biosynthesis}

As carotenoid biosynthesis pathway genes were elevated in TRV-PSY1\&2 (Table 4), the implicated transcription factors among the DEGs may be involved in the regulation of carotenoid biosynthesis; in the upregulated and downregulated DEGs, 40 and 55 transcription factors, respectively, were identified (Additional file 8: Table S7). WRKY, MYB, and NAC were the top three upregulated transcription factor families, whereas ethylene- responsive transcription factor, bHLH, and WRKY were the top three downregulated transcription factor families. This indicates that they may induce the upregulation of carotenoid biosynthesis genes.

\section{Discussion}

Carotenoids play important roles in photosynthesis, hormone signaling, and secondary metabolism. Phytoene synthase is known to play a significant role in the carotenoid biosynthetic pathway owing to its participation in the first committed step and the rate-limiting step, which potentially controls the downstream flux [18]. Even though only one $P S Y$ gene was found in Arabidopsis [19], many plant species are known to have multiple PSY genes with high sequence polymorphisms, including in rice [20], maize [21], and tomato [22-24], indicating a wide functional divergence in the $P S Y$ gene family of plant kingdom; thus, further information is still needed.

\section{PSY gene sequences are highly conserved among Nicotiana species and tomato}

A previous study identified two $P S Y$ genes in N. tabacum using homology-based cloning [36]; however, in tomato, which is also of Solanaceae species, three PSY genes were found [24], suggesting that there may exist some other $P S Y$ genes in tobacco. In this study, we performed a whole genome screening to explore $P S Y$ genes in four Nicotiana species, namely $N$. tabacum, $N$. benthamiana, $N$. sylvestris, and $N$. tomentosiformis; 6,5 , 3 , and 3 PSY genes (Table 1) were identified, respectively. Phylogenetic analysis showed that they can be divided into three groups (Fig. 1). Among them, NtPSY1-1, NtPSY2-1, and NtPSY3-1 were highly correlated with NsylPSY1, NsylPSY2, and NsylPSY3,

Table 3 Statistics of the RNA-Seq reads for TRV-PSY1\&2 and control plants

\begin{tabular}{lllllll}
\hline & TRV_PSY_1 & TRV_PSY_2 & TRV_PSY_3 & Control_1 & Control_2 & Control_3 \\
\hline Raw reads & $42,416,708$ & $59,297,192$ & $39,934,284$ & $56,175,286$ & $40,652,118$ & $38,640,458$ \\
Clean reads & $41,528,460$ & $58,008,450$ & $39,041,364$ & $53,804,542$ & $39,789,524$ & $37,816,924$ \\
Total mapped & $37,172,244(94.11 \%)$ & $34,321,180(94.01 \%)$ & $48,743,371(93.48 \%)$ & $34,281,536(94.28 \%)$ & $36,413,818(94.43 \%)$ & $48,753,836(93.36 \%)$ \\
Mutiple mapped & $31,998,762(81.01 \%)$ & $29,594,117(81.06 \%)$ & $41,808,845(80.18 \%)$ & $29,636,940(81.50 \%)$ & $31,529,268(81.76 \%)$ & $41,082,440(78.67 \%)$ \\
Uniquely mapped & $5,173,482(13.10 \%)$ & $4,727,063(12.95 \%)$ & $6,934,526(13.30 \%)$ & $4,644,596(12.77 \%)$ & $4,884,550(12.67 \%)$ & $7,671,396(14.69 \%)$ \\
\hline
\end{tabular}

TRV_PSY_1, TRV_PSY_2, and TRV_PSY_3 denote the three biological replicates for TRV-PSY1\&2; Control_1, Control_2, and Control_3 are the three biological replicates for the negative control 
Up regulated GO pathways

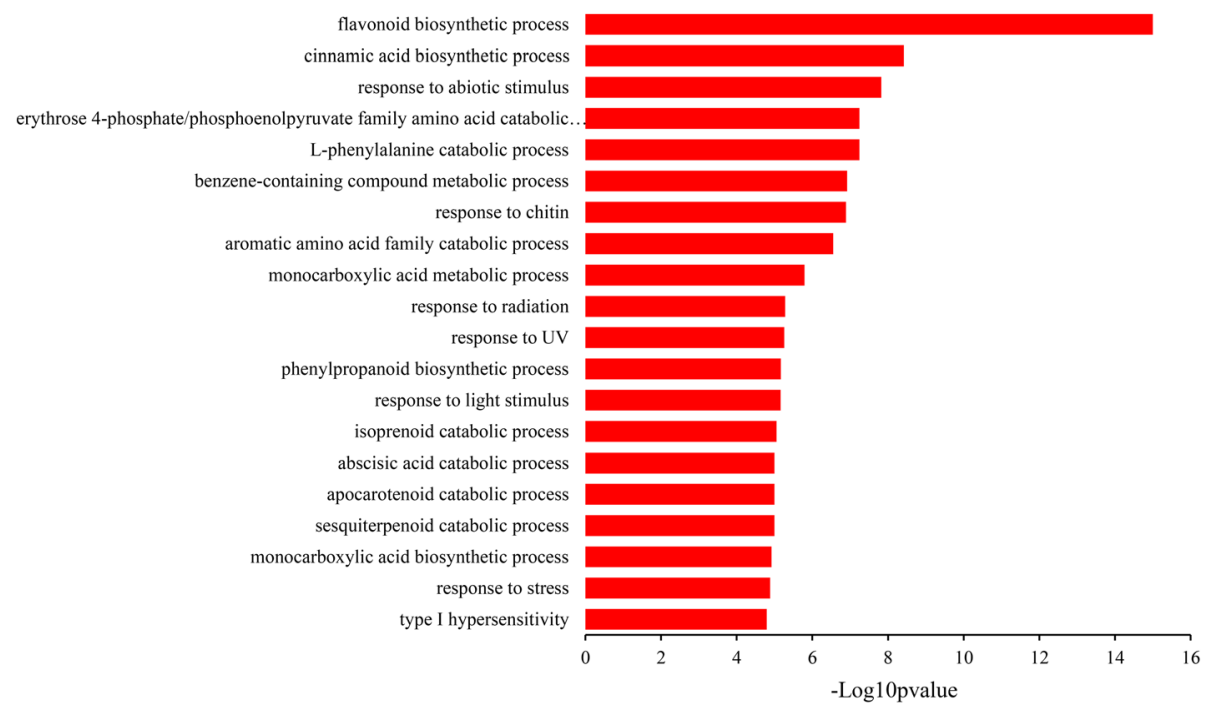

Down regulated GO pathways

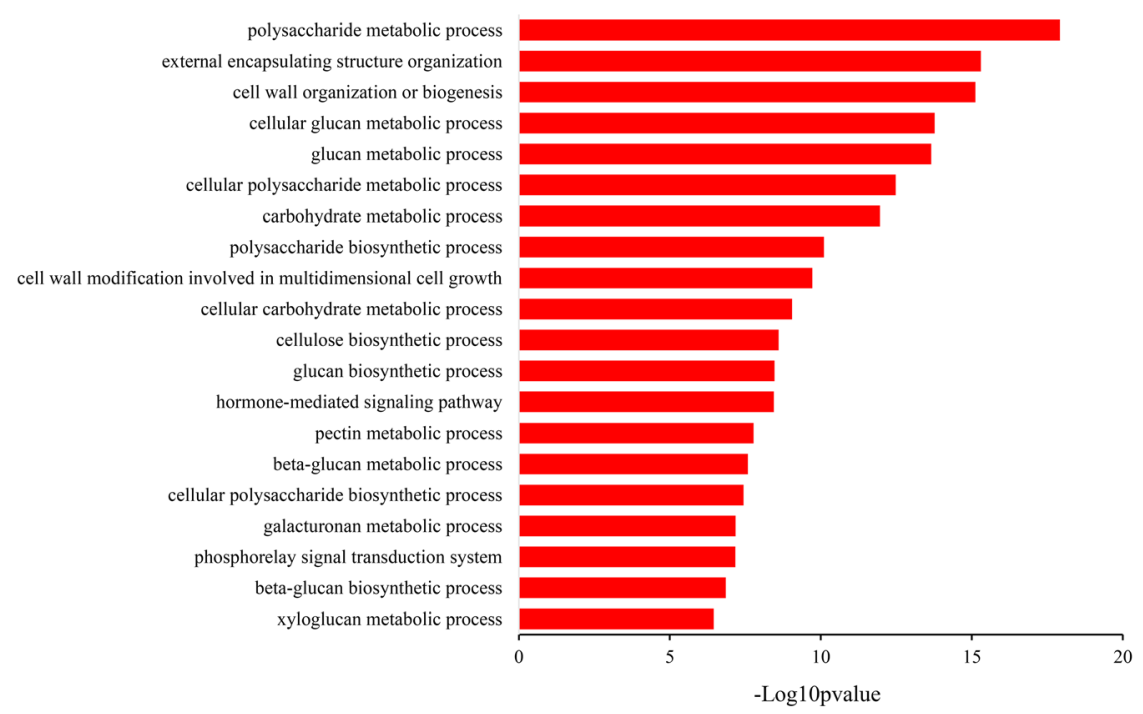

Fig. 6 Gene ontology enrichment analysis for differentially expressed genes between TRV-PSY1\&2 and control plants. Top 20 biological process gene ontology $(\mathrm{GO})$ terms are shown here

respectively. On the other hand, NtPSY1-2, NtPSY2-2, and NtPSY3-2 were clustered more closely with NtomPSY1, NtomPSY2, and NtomPSY3, respectively. Considering that $N$. tabacum is an allotetraploid originating from the hybridization of $N$. sylvestris and $N$. tomentosiformis [37], we speculate that NtPSY1-1, NtPSY2-1, and NtPSY3-1 are derived from N. sylvestris, whereas NtPSY1-2, NtPSY2-2, and NtPSY3-2 originated from $N$. tomentosiformis. $N$. benthamiana PSY genes in each group showed relatively low similarity with those of the other three Nicotiana species, indicating a relatively lower phylogenetic relationship between $N$. benthamiana and other Nicotiana species. Notably, only one PSY2 gene was found in N. benthamiana, which is also an allotetraploid [39]. The lack of the other PSY2 member may be a result of gene loss during polyploidization.

The three PSY genes in tomato were also clustered into three groups (Fig. 1), indicating that tobacco PSY genes are homologs of those in tomato, and PSY gene sequences are conserved in Solanaceae species; however, PSY genes in Arabidopsis, rice, and maize were not clustered with those in tobacco and tomato, and this finding was also reported in a previous study [24], suggesting 
Table 4 Expression levels of carotenoid biosynthesis pathway genes

\begin{tabular}{|c|c|c|c|}
\hline & \multirow[t]{2}{*}{ Gene ID } & \multicolumn{2}{|c|}{ Expression level (TPM) } \\
\hline & & control & TRV-PSY1\&2 \\
\hline \multirow[t]{6}{*}{ GGPPS } & TRINITY_DN36651_C0_g1 & 17.63 & 19.89 \\
\hline & TRINITY_DN35113_c0_g1 & 43.00 & 36.22 \\
\hline & TRINITY_DN69311_C0_g1 & 0.37 & 0.14 \\
\hline & TRINITY_DN37040_c0_g1 & 5.07 & 5.67 \\
\hline & TRINITY_DN40976_c1_g1 & 108.64 & 88.71 \\
\hline & TRINITY_DN39573_c2_g3 & 37.03 & 31.43 \\
\hline \multirow[t]{3}{*}{ PSY } & TRINITY_DN37717_c1_g3 & 19.94 & 6.48 \\
\hline & TRINITY_DN39386_C0_g1 & 106.77 & 60.14 \\
\hline & TRINITY_DN37717_c1_g2 & 62.61 & 26.49 \\
\hline \multirow[t]{3}{*}{ PDS } & TRINITY_DN43680_C0_g1 & 84.11 & 119.76 \\
\hline & TRINITY_DN43680_c0_g4 & 105.50 & 129.32 \\
\hline & TRINITY_DN43680_c0_g3 & 74.50 & 103.00 \\
\hline Z-ISO & TRINITY_DN36957_C0_g1 & 18.62 & 21.02 \\
\hline ZDS & TRINITY_DN38016_c0_g2 & 42.63 & 68.12 \\
\hline CRTISO & TRINITY_DN39955_C0_g1 & 19.45 & 21.73 \\
\hline \multirow[t]{4}{*}{$\beta-L C Y$} & TRINITY_DN38413_c1_g2 & 38.36 & 46.42 \\
\hline & TRINITY_DN33447_c0_g1 & 1.40 & 3.76 \\
\hline & TRINITY_DN33447_c0_g3 & 2.67 & 3.90 \\
\hline & TRINITY_DN33447_c0_g2 & 4.51 & 5.13 \\
\hline \multirow[t]{2}{*}{$\varepsilon-L C Y$} & TRINITY_DN44017_c2_g2 & 27.73 & 47.59 \\
\hline & TRINITY_DN44017_c2_g5 & 43.31 & 70.72 \\
\hline \multirow[t]{3}{*}{$\mathrm{BCH}$} & TRINITY_DN31514_C1_g2 & 1.33 & 1.42 \\
\hline & TRINITY_DN31514_C0_g1 & 6.51 & 18.88 \\
\hline & TRINITY_DN31514_C1_g1 & 1.66 & 1.41 \\
\hline \multirow[t]{3}{*}{ CYP97A3 } & TRINITY_DN41762_C0_g1 & 72.38 & 91.75 \\
\hline & TRINITY_DN41762_c0_g5 & 36.55 & 51.21 \\
\hline & TRINITY_DN41762_C0_g2 & 9.60 & 12.30 \\
\hline \multirow[t]{2}{*}{ CYP97B3 } & TRINITY_DN42530_c2_g4 & 13.25 & 18.58 \\
\hline & TRINITY_DN42530_c2_g2 & 10.87 & 15.26 \\
\hline \multirow[t]{2}{*}{ CYP97C1 } & TRINITY_DN42272_c2_g1 & 25.72 & 30.82 \\
\hline & TRINITY_DN42272_c2_g6 & 43.38 & 41.64 \\
\hline \multirow[t]{2}{*}{ ZEP } & TRINITY_DN42020_c2_g1 & 105.06 & 108.99 \\
\hline & TRINITY_DN42020_c2_g2 & 2.36 & 2.50 \\
\hline \multirow[t]{5}{*}{ NXS } & TRINITY_DN32596_C0_g1 & 12.66 & 11.48 \\
\hline & TRINITY_DN36045_c5_g2 & 11.89 & 7.33 \\
\hline & TRINITY_DN35901_c3_g1 & 12.86 & 5.78 \\
\hline & TRINITY_DN36045_c5_g4 & 8.55 & 8.53 \\
\hline & TRINITY_DN39824_c2_g5 & 0.37 & 0.45 \\
\hline
\end{tabular}

GGPPS, geranylgeranyl diphosphate synthase. PSY, phytoene synthase. PDS, phytoene desaturase. Z-ISO, $\zeta$-isomerase. ZDS, $\zeta$-carotene desaturase. CRTISO, carotenoid isomerase. $\beta$-LCY, lycopene $\beta$-cyclase. $\varepsilon$-LCY, lycopene $\varepsilon$-cyclase. $\mathrm{BCH}$, carotenoid $\beta$-hydroxylase. CYP97A3, cytochrome P450 97A3. CYP97B3, cytochrome P450 97B3. CYP97C1, cytochrome P450 97C1. ZEP, zeaxanthin epoxidase. NXS, neoxanthin synthase. TPM, transcripts per million that the sequences of PSY genes are diverse among different species.

\section{NtPSY1 has a dominant expression pattern relative to other PSY genes}

In our results, tobacco $P S Y 1$ and $P S Y 2$ showed similar expression patterns, with the highest levels in leaves, intermediate in stems and flowers, and low in roots (Fig. $3 a)$, suggesting that they function mainly in aerial tissues. The lack of difference in tissue-specific expression between tobacco PSY1 and PSY2 reduces the possibility of subfunctionalization between them. On the other hand, there may be functional redundancy between tobacco PSY1 and PSY2. PSY1 may have a dominant role in carotenoid biosynthesis, as its expression level is much higher than that of PSY2. This is quite different from tomato, as the three PSY genes work in different tissues, with $P S Y 1$ mainly expressed in fruit [22], $P S Y 2$ works in mature leaves [23], and PSY3 functions in roots [24]. Many studies have found that PSY activity can be regulated at the post-transcriptional level [33, 48-50], which suggests that subfunctionalization between tobacco PSY1 and PSY2 may occur at the protein level; thus, the examination of protein location, catalytic activity, and relative protein content will provide more information about the function of different tobacco PSY genes.

On the other hand, the expression of PSY3 was not detected in any of these tissues (data not shown), indicating that it does not work in these tissues. Tobacco PSY3 belongs to a newly identified $P S Y$ clade, which is widespread but restricted to dicots [24]. Similar to our results, in Manihot esculenta, the PSY3 transcripts were also absent in all the tissues and conditions tested [51]; however, in tomato, PSY3 was strongly expressed exclusively upon root, mainly in response to phosphate starvation, whereas Medicago truncatula PSY3 also works in roots, mainly involved in strigolactones biosynthesis and phosphate starvation [24]. Thus, a possible reason for the lack of expression of tobacco PSY3 is that it is expressed only under special conditions, which is unknown now; however, other possible explanations also exist, for example, it may be a pseudo-gene. In summary, the functions of PSY3 in dicots are far from being well known, and further studies are still needed.

The different expression patterns between tobacco PSY genes may be closely related to their promoter activity, which is supported by the different composition of cis-elements among different genes. As shown in Fig. 2 and Additional file 1: Table S1, some elements such as AE-box, ATCT-motif, TGACT-motif, CGTCA-motif, and GCN4-motif were present only in NtPSY1, which may be responsible for the high transcript levels of NtPSY1. Additionally, some cis-elements such as ACE, 
Box II, GT1-motif, and CAT-box were shared by $N t P S Y 1$ and NtPSY2, but not in NtPSY3, which may explain the non-expression of NtPSY3. The cis-element that was solely present in the NtPSY3 promoter was expected to support the cue for the regulation of NtPSY3. Four cis-elements, that is chs-CMA1a, GATT-motif, LAMP-element, and TGA-element were identified, which were involved in light and auxin response. However, no expression of NtPSY3 was found under light and IAA treatment in our study, suggesting that they were non-functional.

Even though substantial differences were found between the expression levels of different NtPSYs, some cis-elements such as G-box and ABRE were found in all three groups of NtPSY (Additional file 1: Table S1). Some of these elements, such as G-box, play a vital role in light responsive expression of the PSY gene in Arabidopsis [52]. Thus, the different expression patterns of tobacco PSY genes should be regulated by only part of the elements identified in their promoters, and selective deletions of each cis-element are needed to demonstrate their detailed transcriptional regulation mechanism.

\section{Tobacco PSY genes play crucial role in photosynthesis and photoprotection by controlling the synthesis of carotenoids}

Earlier studies have found that carotenoids are essential components of the photosynthetic system, reducing the carotenoid contents will dramatically decrease photosynthetic efficiency, leading to the albino phenotype [1]. The newly emerged leaves of TRV-PSY1\& 2 were also severely blenched (Fig. 4). Based on our results, we speculate that this phenotypic alteration mainly occurred at the metabolic level, but NtPSY was still the causal gene. The direct consequence of NtPSY silencing was the dramatic reduction in carotenoid content (Fig. 5a), which led to the instability of the light-harvesting complex (Fig. $5 \mathrm{~b}$ ) and reduced photosynthetic efficiency (Fig. 5c). In addition, the decline of the NPQ suggests that excess light energy could not be effectively dissipated, which exposed cells to severe oxidative stress [5], eventually leading to cell death and bleach of the leaves.

The consistent results of RNA-Seq and metabolic analysis further strengthen the important role of PSY genes in photosynthesis and photoprotection. Due to the reduction of photosynthetic efficiency, there will be insufficient energy for the cells; thus, many catabolic processes, including amino acids, isoprenoids, and sesquiterpenoids were upregulated in TRV-PSY1\&2 (Fig. 6), consistent with this, metabonomics analysis showed that most of the metabolites were decreased in TRV-PSY1\&2 (Additional file 3: Table S3). GO enrichment also found that pathways response to abiotic stimulus, like radiation, UV and light stimulus were up regulated, besides, flavonoid biosynthetic process was also up regulated, indicated that TRV-PSY1\&2 is suffering severe stress caused by the excess light energy.

The down regulated GO pathways were mainly involved in the biosynthesis of cell wall components, like polysaccharide, glucan, cellulose, pectin and galacturonan (Fig. 6). Metabonomics analysis also showed that some cell wall components were increased in TRVPSY1\&2. More interestingly, the free sedoheptulose was also elevated, sedoheptulose was only liberated in dead plants [42], suggesting that much more cell death occurred in TRV-PSY1\&2, which resulted in the disassembly of cell wall and increase of dissociative components. Similar to our results, it has also been found in tomato that knock down of PSY-1 caused a wide reduction of housekeeping and structural proteins [53].

\section{Tobacco PSY genes are responsive to different phytohormones and light signal}

Previous studies have found that the expression of PSY genes is regulated by various factors, for example, phytohormones such as ethylene and abscisic acid play important roles in the regulation of PSY gene expression. Environmental signals such as strong light, salt, drought, temperature, and photoperiod can also modify the expression level of PSY genes [27]. Transcription factors such as PIF1 and HY5 were found to perceive the signals mentioned above and in turn to control the transcription of PSY genes [31]. In this study, we also identified many cis-elements in PSY gene promoters, most of which were found to respond to the light signals, while phytohormone responsive elements were also found (Fig. 2). Consistent with this, we tested the effects of phytohormones and strong light stress on PSY expression, and found that ABA, 6-BA, and GA treatment could increase the expression of PSY1 and PSY2 (Fig. $3 \mathrm{~b})$. The strong light stress could also elevate PSY1 and PSY2 expression levels (Fig. 3c), indicating that similar to other plant species, tobacco $P S Y 1$ and $P S Y 2$ were regulated by these factors. To our surprise, most ciselements found in $P S Y 1$ and $P S Y 2$ promoters were also present in the PSY3 promoter, but PSY3 showed no response to these treatments we tested, suggesting that PSY3 may work in some other unknown processes.

\section{Tobacco PSYs work synergistically with other genes to control the carotenoids biosynthesis}

As the first enzyme of the carotenoid biosynthesis pathway, PSY has been co-expressed with many photosynthesis-related genes, such as the biosynthesis of carotenoids and chlorophylls [26], which could explain the decrease in chlorophyll content in TRV-PSY1\&2 plants (Fig. 5). Furthermore, in our RNA-Seq analysis, we also found that carotenoid biosynthesis genes were 
coordinated expressed in tobacco, as shown in Table 4. Most of the downstream genes in the carotenoid biosynthesis pathway were upregulated in TRV-PSY1\&2 plants, suggesting that PSY could influence the expression of these genes. Consistent with our results, in tomato transgenic lines that overexpressed PSY-1, most of the downstream genes were suppressed at the transcriptional level [49]. Contrary to the changes in downstream genes, GGPPS, which works up stream of $P S Y$ and is responsible for the precursors of carotenoid biosynthesis, was downregulated in TRV-PSY1\&2 plants (Table 4). Similar to our results, overexpression of tomato PSY-1 elevated the transcript level of GGPPS [49]. Another study found that enhanced PSY activity could upregulate DXS levels [18]. DXS is an MEP pathway enzyme that also works up stream of PSY and response for the biosynthesis of isoprenoids, indicating that changes in PSY level could also influence the expression of the upstream genes. In tomato, PSY could be associated with other enzymes such as GGPPS into large protein complexes [48], suggesting that this association may influence the coregulation of these genes.

Previous studies have identified a common ATCTAmotif in the promoter of some carotenoid biosynthesis genes, including $P S Y$ and $P D S$, and their upstream genes $D X S$ and HDR in the MEP pathway. This motif is a binding site of ERF transcription factor [52, 54]. In this study, we identified 95 transcription factors among the DEGs (Additional file 8: Table S7). Among them, 15 belonged to the ERF family, indicating that they may be involved in the regulation of the coordinated expression between carotenoid biosynthesis genes, which needs further verification.

\section{Conclusions}

We identified three groups of PSY genes in four Nicotiana species, which shared high similarity with those in tomato, but not with those in monocots. PSY1 and PSY2 showed the highest expression levels in leaves, and could be elevated by phytohormones and strong light treatment, but no expression of PSY3 was detected. The photosynthetic system activity were significantly decreased in PSY1 and PSY2 silencing plants. RNA-Seq analysis showed that tobacco PSYs work synergistically with other genes to control carotenoid biosynthesis. The information obtained here may aid further research on PSY genes and carotenoid biosynthesis.

\section{Methods}

\section{Plant materials and growth conditions}

Nicotiana benthamiana and common tobacco (Nicotiana tabacum L.) variety K326 were used in this study. Seeds were germinated on moist soil and grown under $16 \mathrm{~h}$ light, $8 \mathrm{~h}$ dark, and $25^{\circ} \mathrm{C}$ conditions.

\section{Identification of PSY genes in tobacco genomes}

The genome sequences and annotation information of K326, Nicotiana benthamiana, Nicotiana sylvestris, and Nicotiana tomentosiformis were obtained from Sol Genomics Network (SGN) database (https://solgenomics.net/). The Arabidopsis PSY protein sequence (At5g17230) was obtained from the The Arabidopsis Information Resource (TAIR) database (https://www.arabidopsis.org/) and used as a query sequence to screen PSY sequences in various tobacco species using BlastP program and e-value $<1 \mathrm{e}^{-10}$ as the query threshold. A PSY domain (accession PF00494) was extracted from Pfam database (http://pfam. xfam.org/) to determine PSY sequences using HMMER web server (https://www.ebi.ac.uk/Tools/hmmer/) [55].

\section{Phylogenetic analysis}

To elucidate the phylogenetic relationship between tobacco PSY proteins and those of other species, phylogenetic analysis was conducted using MEGA 5 software [56]. The sequences and corresponding sequence accession numbers of PSY proteins in Arabidopsis, rice, maize, and tomato were used as previously described [24] and downloaded from GeneBank database (https:// www.ncbi.nlm.nih.gov/).

Multiple sequence alignments of amino acid sequences were performed using the CLUSTALW algorithm using default parameters, and the resulting aligned region was used for phylogenetic analysis by Neighbor-Joining method [57], and the phylogenetic tree was constructed with 1000 bootstrap replicates.

\section{Cis-element analysis of tobacco NtPSY gene promoters}

The $2000 \mathrm{bp}$ sequence upstream of the start codons of NtPSY genes was obtained from the SGN database, and cis-element analysis was performed using PlantCARE web tools (http://bioinformatics.psb.ugent.be/ webtools/plantcare/html/). The results obtained were visualized using of GSDS2.0 web server (http://gsds. cbi.pku.edu.cn/).

\section{Treatment with phytohormones and exposure to strong light}

At the fifth-leaf stage, K326 plants were separately sprayed with $50 \mu \mathrm{mol} / \mathrm{L}$ gibberellic acid (GA), $100 \mu \mathrm{mol} /$ $\mathrm{L}$ methyl jasmonate (MeJA), $10 \mu \mathrm{mol} / \mathrm{L}$ abscisic acid (ABA), $2 \mu \mathrm{mol} / \mathrm{L}$ 6-benzyladenine (6-BA), or $5 \mu \mathrm{mol} / \mathrm{L} 3-$ indoleacetic acid (IAA), the control plants were sprayed with double distilled water; leaves were harvested $8 \mathrm{~h}$ after treatment. Strong light conditions was defined as $1200 \mu \mathrm{mol} \cdot \mathrm{m}^{-2} \cdot \mathrm{s}^{-1}$ and the control conditions as $400 \mu \mathrm{mol} \cdot \mathrm{m}^{-2} \cdot \mathrm{s}^{-1}$, and samples were harvested at $0 \mathrm{~h}$ and, 1, 2, and $4 \mathrm{~h}$ after treatment. Three biological replicates were used for each treatment. The harvested 
materials were immediately submerged under liquid nitrogen and stored at $-80^{\circ} \mathrm{C}$ until use.

\section{Virus-induced NbibenPSY silencing}

To elucidate the biological functions of tobacco PSY, NbibenPSY genes were silenced using virus-induced gene silencing methods. A 684-bp cDNA fragment, which showed $96.2 \%$ similarity with NbibenPSY1-1 and NbibenPSY1-2, was selected for simultaneous gene silencing. Another cDNA fragment of $726 \mathrm{bp}$ was selected for NbibenPSY2 silencing. The two fragments were obtained by PCR amplification using a template of leaf cDNA. The primers used are listed in Additional file 2: Table S2, with the restriction sites of Kpn I and Xho I as the cloning sites for forward and reverse primers, respectively.

The two fragments and the empty pTRV2 (pYL156) vector (described in Liu et al., [58]) were digested separately using Kpn I and Xho I restriction enzymes. Then, the fragments were ligated into digested pYL156 vectors, and confirmed by sequencing. Thus two constructs were obtained, namely TRV-PSY1 and TRV-PSY2. The two constructs were then transferred into Agrobacterium tumefaciens strain GV3101 using freeze-thaw method.

The infiltration of $N$. benthamiana leaves was performed mainly based on previously described methods [59]. Briefly, A. tumefaciens strains containing TRVPSY1 or TRV-PSY2 were grown at $28^{\circ} \mathrm{C}$ in Luria Bertani (LB) medium containing appropriate antibiotics. The cells were harvested and resuspended in the infiltration buffer $(10 \mathrm{~mm}$ MES, $\mathrm{pH}=5.5,200 \mu \mathrm{m}$ acetosyringone, and $10 \mathrm{mM} \mathrm{MgCl}$ ) to a final absorbance (optical density (OD) at $600 \mathrm{~nm}$ ) of 1.0 and incubated for $2 \mathrm{~h}$ at $25 \pm$ $2{ }^{\circ} \mathrm{C}$. For leaf infiltration, each $A$. tumefaciens strain containing TRV-PSY1 or TRV-PSY2 were mixed in a 1:1 ratio in infiltration buffer and infiltrated into lower leaves using a $1 \mathrm{ml}$ needleless syringe. The empty pYL156 vector and its derivative, TRV-PDS construct (could silence the phytoene desaturase gene as described previously [41]) were used as negative and positive controls, respectively, using the same method. The infiltrated plants were maintained at $25^{\circ} \mathrm{C}$ for effective viral infection and spread.

\section{Photosynthetic activity measurement}

The isolation of carotenoid and chlorophyll was performed as previously described [60]. Briefly, $50 \mathrm{mg}$ (fresh weight) samples were mixed and shook with $1 \mathrm{ml} 80 \%$ (v/v) ice-cold acetone in the dark at $4^{\circ} \mathrm{C}$ for $30 \mathrm{~min}$. After centrifugation $\left(10,000 \times \mathrm{g}, 2 \mathrm{~min}, 4^{\circ} \mathrm{C}\right)$, absorbances at $663 \mathrm{~nm}, 647 \mathrm{~nm}$, and $470 \mathrm{~nm}$ were recorded using a spectrophotometer, and pigment levels were calculated using the following equation: chlorophyll $\mathrm{a}=$ 12.25*A663-2.79*A647; chlorophyll $\mathrm{b}=21.50 * \mathrm{~A} 647-$
5.10*A663; chlorophyll total $=7.15^{*} \mathrm{~A} 663+18.71^{*} \mathrm{~A} 647$; carotenoids $=\left(1000^{*} \mathrm{~A} 470-1.82^{*}\right.$ chlorophyll a $-85.02^{*}$ chlorophyll b)/198.

Chlorophyll fluorescence was measured using DualPAM 100 (WALZ, Germany); four parameters, namely $\mathrm{Fv} / \mathrm{Fm}$ (maximum quantum efficiency of PSII photochemistry), ФPSII (sum of the quantum yields of PSII photochemistry), qP (photochemical quenching), and NPQ (non-photochemical quenching) were measured.

For thylakoid isolation, $1 \mathrm{~g}$ samples was put in $5 \mathrm{ml}$ extracting buffer $(500 \mathrm{mM}$ sorbitol, $50 \mathrm{mM}$ Tris- $\mathrm{HCl}, 2$ mM EDTA, $1 \mathrm{mM} \mathrm{MgCl}$, and $1 \mathrm{mM} \mathrm{MnCl}_{2}, \mathrm{pH}=6.8$, $\left.4{ }^{\circ} \mathrm{C}\right)$ ground, and filtered through a cell filter. Then, the mixture was centrifuged at $4{ }^{\circ} \mathrm{C}, 8000 \mathrm{~g}$ for $5 \mathrm{~min}$. The thylakoids in the supernatant were then washed using 25BTH20G buffer (pH $=7.0,20 \%$ glycerol, and $25 \mathrm{mM}$ Bis-Tris), and centrifuged at $4{ }^{\circ} \mathrm{C}, 15,000 \times \mathrm{g}$ for $5 \mathrm{~min}$. Blue-native polyacrylamide gel electrophoresis (BNPAGE) was performed as previously described [61] with some modifications; $12 \mu \mathrm{g}$ chlorophyll was incubated with $1 \% \beta$-DM, and the solubilized fraction was then loaded on a native gradient gel $(5-12 \%(w / v)$, acrylamide/bisacrylamide ratio 32:1) topped with a $4 \%(\mathrm{w} / \mathrm{v})$ stacking gel (ratio 1:4). After electrophoresis, the native gel was treated for $1.5 \mathrm{~h}$ with Laemmli buffer $(138 \mathrm{mM}$ Tris- $\mathrm{HCl}$ [pH 6.8], $6 \mathrm{M}$ urea, 22.2\% [v/v] glycerol, $4.3 \%$

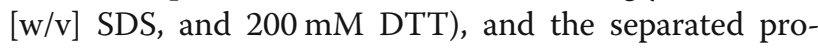
tein complexes were transferred onto a polyvinylidene fluoride membrane using Turbo Transfer system (BioRad).

\section{Leaf metabolomics analysis}

The metabolic profile of tobacco leaves from control and TRV-PSY1\&2 was investigated using gas chromatography-mass spectrometry (GC-MS) according to previously described methods [62] with some modifications. The freeze-dried tissue was ground to a uniform powder and filtered using a 40-mesh sieve. Leaf powder $(10 \mathrm{mg}$ ) was added to a $2 \mathrm{ml}$ Eppendorf tube and soaked in $1.5 \mathrm{ml}$ extraction solvent containing isopropanol/ acetonitrile/water $(3 / 3 / 2, \mathrm{v} / \mathrm{v} / \mathrm{v})$ with $25 \mu \mathrm{l}(0.1 \mathrm{mg} / \mathrm{ml})$ tridecanoic acid as an internal standard. All extracts were sonicated for $1 \mathrm{~h}$ and centrifuged for $10 \mathrm{~min}$ (14, $000 \mathrm{rpm}, 4{ }^{\circ} \mathrm{C}$ ). Four-hundred $\mu \mathrm{l}$ the supernatant was transferred to a new tube and dried under nitrogen flow on an N-EVAP nitrogen evaporator. To increase the volatility of the metabolites, silylation reaction was performed by adding $100 \mu \mathrm{l}$ methyl-trimethyl-silyl-trifluoroacetamide (MSTFA) to the sample and incubating it for $60 \mathrm{~min}$ at $60^{\circ} \mathrm{C}$.

GC-MS analysis of the metabolomic analysis was performed on Agilent 7683B series injector (Agilent, Santa Clara, CA) coupled to an Agilent $6890 \mathrm{~N}$ series gas chromatography system and 5975 mass selective detector 
(MSD) (Agilent, Santa Clara, CA). Agilent DB-5MS column $(0.25 \mu \mathrm{m}, 0.25 \mathrm{~mm} \times 30 \mathrm{~m}$, Agilent Technologies, Inc., Santa Clara, CA) was used. The column temperature was set at $70^{\circ} \mathrm{C}$ for the first $4 \mathrm{~min}$ and then increased at $5{ }^{\circ} \mathrm{C} / \mathrm{min}$ to $310^{\circ} \mathrm{C}$ for $15 \mathrm{~min}$. Helium (99.9995\%) was used as the carrier gas. The column flow was $1.2 \mathrm{ml} / \mathrm{min}$, and the column was equipped with a linear velocity control model. The temperatures of the interface and the ion source were adjusted to $280^{\circ} \mathrm{C}$ and $230^{\circ} \mathrm{C}$, respectively. The electron impact (EI) model was set to achieve ionization of the metabolites at $70 \mathrm{eV}$.

Student's $t$-test was used to determine the significant differences between the metabolites in the control and TRV-PSY1\&2 (SPSS software, version 17.0).

\section{RNA preparation}

Different plant tissues were harvested and immediately frozen using liquid nitrogen. Total RNA was isolated using Spin Column Plant Total RNA Purification Kit (Sangon Biotech, China). The quality and quantity of these RNA samples were further determined using Nanodrop 2000 (Thermo Fisher, US) and agarose gel electrophoresis.

\section{Reverse transcription and quantitative real-time PCR (qPCR) analysis}

First-strand cDNA was synthesized using PrimeScript reverse transcriptase (Takara). The primers used for qPCR are listed in Additional file 2: Table S2. qPCR was performed using Roche Light-Cycler 480 System. The reaction mixture contained $2 \mu \mathrm{l}$ primers $(2.5 \mu \mathrm{M}), 10 \mu \mathrm{l}$ SYBR Green I Master Mix (Roche), $2 \mu$ cDNA template, and $6 \mu \mathrm{l}$ water. The real-time PCR conditions were set as follows: $95^{\circ} \mathrm{C}$ for $5 \mathrm{~min}$, followed by 45 cycles of $95^{\circ} \mathrm{C}$ for $10 \mathrm{~s}, 60^{\circ} \mathrm{C}$ for $30 \mathrm{~s}$, and $72{ }^{\circ} \mathrm{C}$ for $20 \mathrm{~s}$. A melting curve was established by slow heating from $60^{\circ} \mathrm{C}$ to $95^{\circ} \mathrm{C}$ throughout $20 \mathrm{~min}$. Relative gene expression levels were calculated using $2^{-\Delta \Delta C T}$ with three replicates for each sample. Data are presented as means \pm standard deviation (SD) $(n=3)$.

\section{RNA-seq and data processing}

Six samples were used for the RNA-seq analyses: three from the TRV-PSY1 and TRV-PSY2 co-infiltrate plants (TRV-PSY1\&2) and three from the control plants. Total RNA was sent to Sangon Biotech (Shanghai) Co., Ltd., where the libraries were produced. The cDNA libraries were then sequenced using the Illumina HiSeq 2000. Then, 150-bp paired-end clean data were obtained by excluding the adaptors and low-quality reads using Trimmomatic software [63]. The clean reads generated were processed using Trinity software [43] to assemble a de novo transcriptome and used as a reference sequence for downstream analysis.
Unigenes obtained from the de novo transcriptome were queried against and annotated using the following databases: NT (NCBI nucleotide sequences), NR (NCBI non-redundant protein sequences), COG (Clusters of Orthologous Groups of proteins), KOG (euKaryotic Ortholog Groups), Swiss-Prot (A manually annotated and reviewed protein sequence database), TrEMBL, PFAM (Protein family), CDD (Conserved Domain Database), GO (Gene Ontology), and KEGG (Kyoto Encyclopedia of Genes and Genomes).

The set of unigenes obtained above was used as a reference sequence, and clean reads of each sample were then mapped to the sequence using Bowtie2 software [44]. Gene expression levels were calculated based on the reads mapping results and shown as transcripts per million (TPM) value [45].

We used the DESeq software [46] to identify differentially expressed genes (DEGs) between samples. An adjusted $p$-value $<0.05$ found by DESeq were applied as standards to characterize the significance of gene expression levels. To identify the pathways significantly affected by the PSY genes, GO enrichment pathway analysis of DEGs was performed using topGO software [47].

\section{Supplementary Information}

The online version contains supplementary material available at https://doi. org/10.1186/s12870-020-02816-3.

Additional file 1: Table S1.docx Cis-regulatory elements found in the promoter region of NTPSY genes.

Additional file 2: Table S2.docx Primer sequences used in $\mathrm{qPCR}$ analysis. The underlined letters indicate the manually added cloning site adaptors: Kpn I and Xho I for forward and reverse primers, respectively.

Additional file 3: Table S3.xIsx Relative metabolite levels in negative control and TRV-PSY1\&2 leaves.

Additional file 4: Table S4.docx Summary of unigene annotation. Additional file 5: Table S5.xlsx Differentially expressed genes between TRV-PSY1\&2 and control plants

Additional file 6: Table S6.xlsx Gene ontology enrichment of differentially expressed genes between TRV-PSY1\&2 and control plants

Additional file 7: Figure S1.tif qRT-PCR confirmation of carotenoid biosynthesis genes in TRV-PSY1\&2 and control plants. GGPPS, geranylgeranyl diphosphate synthase. PDS, phytoene desaturase. ZDS, Z-carotene desaturase. CRTISO, carotenoid isomerase. B-LCY, lycopene $\beta$-cyclase. NXS, neoxanthin synthase. Columns and bars represent the means and standard errors $(n=3)$, respectively. ${ }^{*}$ indicates $P<0.05$.

Additional file 8: Table S7.xlsx Differentially expressed transcription factors between TRV-PSY1\&2 and control plants

\section{Abbreviations}

ABA: Abscisic acid; BN-PAGE: Blue-native polyacrylamide gel-electrophoresis; DEG: Differentially Expressed Gene; GA: Gibberellin; GC-MS: Gas

chromatography-mass spectrometry; GGPP: Geranylgeranyl diphosphate; IAA: Indole-3-acetic acid; MeJA: Methyl Jasmonate; PSY: Phytoene synthase; 6-BA: 6-benzyladenine

Acknowledgements

Not applicable. 


\section{Authors' contributions}

ZJ.W., L.Z., finished the most of experiments and data analysis. C.D., JG.G., created the plant lines and did the photosynthetic experiments. LF.J., P.W., F.L., did the plant cultivation and pigment content anlysis. XQ.Z., R.W., design the project. ZJ.W., XQ.Z., R.W., wrote the manuscript. All authors have read and approved the manuscript.

\section{Funding}

This research was funded by grants from the Natural Science Foundation of Henan Province (182300410053/902018AS0010 to RW), the Tobacco Genome Project 902018AA0120.2/902019AA0140, the key science and technology research project of Henan Province (202102110023), the 111 Project\#D16014. The funding bodies played no role in the design of the study and collection, analysis, and interpretation of data and in writing the manuscript.

\section{Availability of data and materials}

The RNA-seq datasets used this article are available in the NCBI Sequence Read Archive (SRA) (https://www.ncbi.nlm.nih.gov/sra/) under BioProject accession: PRJNA631583. The data that support the results are included within the article and its additional files.

\section{Ethics approval and consent to participate} Not applicable.

\section{Consent for publication}

Not applicable.

\section{Competing interests}

The authors declare that they have no competing interests.

\section{Author details}

${ }^{1}$ College of Tobacco Science, Henan Agricultural University, Zhengzhou 450002, China. ${ }^{2}$ China Tobacco Gene Research Center, Zhengzhou Tobacco Research Institute, Zhengzhou 450001, China. ${ }^{3}$ China Tobacco Yunnan Industrial Co., Ltd., Kunming 650231, Yunnan, China. ${ }^{4}$ State Key Laboratory of Cotton Biology, Key Laboratory of Plant Stress Biology, School of Life Sciences, Henan University, 85 Minglun Street, Kaifeng 475001, China. ${ }^{5}$ School of Life Sciences, School of Agricultural Sciences, Zhengzhou University, No. 100 Science Road, Gaoxin Distract, Zhengzhou 450001, Henan, China.

Received: 17 July 2020 Accepted: 22 December 2020

Published online: 07 January 2021

\section{References}

1. Rodriguez-Concepcion M, Avalos J, Bonet ML, Boronat A, Gomez-Gomez L, Hornero-Mendez D, Limon MC, Melendez-Martinez AJ, OlmedillaAlonso B, Palou A, et al. A global perspective on carotenoids: metabolism, biotechnology, and benefits for nutrition and health. Prog Lipid Res. 2018;70:62-93.

2. Sun T, Li L. Toward the 'golden' era: the status in uncovering the regulatory control of carotenoid accumulation in plants. Plant Sci. 2020;290:110331.

3. Yabuzaki J. Carotenoids Database: structures, chemical fingerprints and distribution among organisms. Database. 2017:bax004.

4. Eggersdorfer $M$, Wyss $A$. Carotenoids in human nutrition and health. Arch Biochem Biophys. 2018;652:18-26.

5. Peñuelas J, Munné-Bosch S. Isoprenoids: an evolutionary pool for photoprotection. Trends Plant Sci. 2005:10(4):166-9.

6. Hashimoto H, Uragami C, Cogdell RJ. Carotenoids and photosynthesis. In: Stange $C$, editor. Carotenoids in nature: biosynthesis, regulation and function. Cham: Springer International Publishing; 2016. p. 111-39.

7. Finkelstein R. Abscisic acid synthesis and response. Arabidop Book. 2013:11:e116.

8. Jia KP, Baz L, Al-Babili S. From carotenoids to strigolactones. J Exp Bot. 2018; 69(9):2189-204.

9. Britton G. Functions of intact carotenoids. In: Britton G, Liaaen-Jensen S, Pfander $\mathrm{H}$, editors. Carotenoids: volume 4: natural functions. Basel: Birkhäuser Basel; 2008. p. 189-212.

10. Vranova E, Coman D, Gruissem W. Network analysis of the MVA and MEP pathways for isoprenoid synthesis. Annu Rev Plant Biol. 2013;64:665-700.
11. Ruiz-Sola MA, Rodriguez-Concepcion M. Carotenoid biosynthesis in Arabidopsis: a colorful pathway. Arabidop Book. 2012;10:e0158.

12. Qin G, Gu H, Ma L, Peng Y, Deng XW, Chen Z, Qu L-J. Disruption of phytoene desaturase gene results in albino and dwarf phenotypes in Arabidopsis by impairing chlorophyll, carotenoid, and gibberellin biosynthesis. Cell Res. 2007;17(5):471-82.

13. Dong H, Deng Y, Mu J, Lu Q, Wang Y, Xu Y, Chu C, Chong K, Lu C, Zuo J. The Arabidopsis Spontaneous Cell Death1 gene, encoding a ל-carotene desaturase essential for carotenoid biosynthesis, is involved in chloroplast development, photoprotection and retrograde signalling. Cell Res. 2007; 17(5):458-70

14. Chen Y, Li F, Wurtzel ET. Isolation and characterization of the Z-ISO gene encoding a missing component of carotenoid biosynthesis in plants. Plant Physiol. 2010;153(1):66

15. Cunningham FX, Gantt E. One ring or two? Determination of ring number in carotenoids by lycopene $\varepsilon$-cyclases. P Natl Acd Sci USA. 2001;98(5):2905

16. Pecker I, Gabbay R, Cunningham FX, Hirschberg J. Cloning and characterization of the cDNA for lycopene $\beta$-cyclase from tomato reveals decrease in its expression during fruit ripening. Plant Mol Biol. 1996;30(4): 807-19.

17. Kim J, Smith JJ, Tian L, DellaPenna D. The evolution and function of carotenoid hydroxylases in Arabidopsis. Plant Cell Physiol. 2009;50(3):463-79.

18. Rodríguez-Villalón A, Gas E, Rodríguez-Concepción M. Phytoene synthase activity controls the biosynthesis of carotenoids and the supply of their metabolic precursors in dark-grown Arabidopsis seedlings. Plant J. 2009; 60(3):424-35.

19. Scolnik PA, Bartley GE. Nucleotide sequence of an Arabidopsis CDNA for phytoene synthase. Plant Physiol. 1994;104(4):1471-2.

20. Welsch R, Wüst F, Bär C, Al-Babili S, Beyer P. A third phytoene synthase is devoted to abiotic stress-induced abscisic acid formation in rice and defines functional diversification of phytoene synthase genes. Plant Physiol. 2008; 147(1):367.

21. Li F, Vallabhaneni R, Yu J, Rocheford T, Wurtzel ET. The maize phytoene synthase gene family: overlapping roles for carotenogenesis in endosperm, photomorphogenesis, and thermal stress tolerance. Plant Physiol. 2008; 147(3):1334.

22. Bartley GE, Viitanen PV, Bacot KO, Scolnik PA. A tomato gene expressed during fruit ripening encodes an enzyme of the carotenoid biosynthesis pathway. J Biol Chem. 1992;267(8):5036-9.

23. Bartley GE, Scolnik PA. cDNA cloning, expression during development, and genome mapping of PSY2, a second tomato gene encoding phytoene synthase. J Biol Chem. 1993;268(34):25718-21.

24. Stauder R, Welsch R, Camagna M, Kohlen W, Balcke GU, Tissier A, Walter MH Strigolactone levels in dicot roots are determined by an ancestral symbiosisregulated clade of the PHYTOENE SYNTHASE gene family. Front Plant Sci. 2018;9:255.

25. Li F, Vallabhaneni $R$, Wurtzel ET. PSY3, a new member of the phytoene synthase gene family conserved in the Poaceae and regulator of abiotic stress-induced root carotenogenesis. Plant Physiol. 2008;146(3):1333.

26. Meier S, Tzfadia O, Vallabhaneni R, Gehring C, Wurtzel ET. A transcriptional analysis of carotenoid, chlorophyll and plastidial isoprenoid biosynthesis genes during development and osmotic stress responses in Arabidopsis thaliana. BMC Syst Biol. 2011;5(1):77.

27. Nisar N, Li L, Lu S, Khin Nay C, Pogson BJ. Carotenoid metabolism in plants. Mol Plant. 2015:8(1):68-82.

28. Marty I, Bureau S, Sarkissian G, Gouble B, Audergon JM, Albagnac G. Ethylene regulation of carotenoid accumulation and carotenogenic gene expression in colour-contrasted apricot varieties (Prunus armeniaca). J Exp Bot. 2005;56(417):1877-86.

29. Cazzonelli Cl, Pogson BJ. Source to sink: regulation of carotenoid biosynthesis in plants. Trends Plant Sci. 2010;15(5):266-74.

30. Toledo-Ortiz G, Huq E, Rodríguez-Concepción M. Direct regulation of phytoene synthase gene expression and carotenoid biosynthesis by phytochrome-interacting factors. P Natl Acd Sci USA. 2010;107(25):11626.

31. Toledo-Ortiz G, Johansson H, Lee KP, Bou-Torrent J, Stewart K, Steel G, Rodríguez-Concepción M, Halliday KJ. The HY5-PIF regulatory module coordinates light and temperature control of photosynthetic gene transcription. PLoS Genet. 2014:10(6):e1004416.

32. Shumskaya M, Bradbury LMT, Monaco RR, Wurtzel ET. Plastid localization of the key carotenoid enzyme phytoene synthase is altered by isozyme, allelic variation, and activity. Plant Cell. 2012;24(9):3725. 
33. Zhou X, Welsch R, Yang Y, Álvarez D, Riediger M, Yuan H, Fish T, Liu J, Thannhauser TW, Li L. Arabidopsis OR proteins are the major posttranscriptional regulators of phytoene synthase in controlling carotenoid biosynthesis. P Natl Acd Sci USA. 2015;112(11):3558.

34. Zhao Q, Wang G, Ji J, Jin C, Wu W, Zhao J. Over-expression of Arabidopsis thaliana $\beta$-carotene hydroxylase (chyB) gene enhances drought tolerance in transgenic tobacco. J Plant Biochem Biot. 2014;23(2):190-8.

35. Mann V, Harker M, Pecker I, Hirschberg J. Metabolic engineering of astaxanthin production in tobacco flowers. Nat Biotechnol. 2000;18(8):888-92.

36. Busch M, Seuter A, Hain R. Functional analysis of the early steps of carotenoid biosynthesis in tobacco. Plant Physiol. 2002;128(2):439.

37. Sierro N, Battey JND, Ouadi S, Bakaher N, Bovet L, Willig A, Goepfert S, Peitsch MC, Ivanov NV. The tobacco genome sequence and its comparison with those of tomato and potato. Nat Commun. 2014:5.

38. Edwards KD, Fernandez-Pozo N, Drake-Stowe K, Humphry M, Evans AD, Bombarely A, Allen F, Hurst R, White B, Kernodle SP, et al. A reference genome for Nicotiana tabacum enables map-based cloning of homeologous loci implicated in nitrogen utilization efficiency. BMC Genomics. 2017:18.

39. Bombarely A, Rosli HG, Vrebalov J, Moffett P, Mueller LA, Martin GB. A draft genome sequence of Nicotiana benthamiana to enhance molecular plantmicrobe biology research. Mol Plant Microbe. 2012;25(12):1523-30.

40. Sierro N, Battey JND, Ouadi S, Bovet L, Goepfert S, Bakaher N, Peitsch MC, Ivanov NV. Reference genomes and transcriptomes of Nicotiana sylvestris and Nicotiana tomentosiformis. Genome Biol. 2013;14(6):R60.

41. Liu E, Page JE. Optimized cDNA libraries for virus-induced gene silencing (VIGS) using tobacco rattle virus. Plant Methods. 2008;4(1):5.

42. Benson AA, Bassham JA, Calvin M. Sedoheptulose in photosynthesis by plants. J Am Chem Soc. 1951;73(6):2970.

43. Grabherr MG, Haas BJ, Yassour M, Levin JZ, Thompson DA, Amit I, Adiconis $X$, Fan L, Raychowdhury R, Zeng Q, et al. Full-length transcriptome assembly from RNA-Seq data without a reference genome. Nat Biotechnol. 2011;29(7): 644-52.

44. Langmead B, Salzberg SL. Fast gapped-read alignment with bowtie 2. Nat Meth. 2012;9(4):357-9.

45. Wagner GP, Kin K, Lynch VJ. Measurement of mRNA abundance using RNAseq data: RPKM measure is inconsistent among samples. Theor Biosci. 2012; 131(4):281-5.

46. Anders $\mathrm{S}$, Huber W. Differential expression of RNA-Seq data at the gene level-the DESeq package. European Molecular Biology Laboratory: Heidelberg; 2012. Available at www.bioconductor.org/packages/devel/bioc/ vignettes/DESeq/inst/doc/DESeq.pdf.

47. Alexa A, Rahnenfuhrer J. topGO: enrichment analysis for gene ontology. R package version. 2010. Available at http://www.bioconductor.org/ packages/2.11/bioc/html/topGO.html.

48. Fraser PD, Schuch W, Bramley PM. Phytoene synthase from tomato (Lycopersicon esculentum) chloroplasts - partial purification and biochemical properties. Planta. 2000;211(3):361-9.

49. Fraser PD, Enfissi EMA, Halket JM, Truesdale MR, Yu D, Gerrish C, Bramley PM. Manipulation of phytoene levels in tomato fruit: effects on isoprenoids, plastids, and intermediary metabolism. Plant Cell. 2007;19(10):3194-211.

50. Álvarez D, Voß B, Maass D, Wüst F, Schaub P, Beyer P, Welsch R. Carotenogenesis is regulated by $5^{\prime} \mathrm{UTR}$-mediated translation of phytoene synthase splice variants. Plant Physiol. 2016;172(4):2314-26.

51. Arango J, Wüst F, Beyer P, Welsch R. Characterization of phytoene synthases from cassava and their involvement in abiotic stress-mediated responses. Planta. 2010;232(5):1251-62.

52. Welsch R, Medina J, Giuliano G, Beyer P, von Lintig J. Structural and functional characterization of the phytoene synthase promoter from Arabidopsis thaliana. Planta. 2003;216(3):523-34.

53. Robertson FP, Koistinen PK, Gerrish C, Halket JM, Patel RKP, Fraser PD, Bramley PM. Proteome changes in tomato lines transformed with phytoene synthase- 1 in the sense and antisense orientations. J Exp Bot. 2012;63(16):6035-43.

54. Botella-Pavía P, Besumbes Ó, Phillips MA, Carretero-Paulet L, Boronat A, Rodríguez-Concepción M. Regulation of carotenoid biosynthesis in plants: evidence for a key role of hydroxymethylbutenyl diphosphate reductase in controlling the supply of plastidial isoprenoid precursors. Plant J. 2004;40(2):188-99.

55. Finn RD, Clements J, Eddy SR. HMMER web server: interactive sequence similarity searching. Nucleic acids Res. 2011;39(suppl 2):W29-37.
56. Tamura K, Peterson D, Peterson N, Stecher G, Nei M, Kumar S. MEGA5 molecular evolutionary genetics analysis using maximum likelihood, evolutionary distance, and maximum parsimony methods. Mol Biol Evol. 2011:28(10):2731-9.

57. Saitou N, Nei M. The neighbor-joining method: a new method for reconstructing phylogenetic trees. Mol Biol Evol. 1987;4(4):406-25.

58. Liu Y, Schiff M, Marathe R, Dinesh-Kumar SP. Tobacco Rar1, EDS1 and NPR1/ NIM1 like genes are required for $\mathrm{N}$-mediated resistance to tobacco mosaic virus. Plant J. 2002;30(4):415-29.

59. Senthil-Kumar M, Hema R, Anand A, Kang L, Udayakumar M, Mysore KS. A systematic study to determine the extent of gene silencing in Nicotiana benthamiana and other Solanaceae species when heterologous gene sequences are used for virus-induced gene silencing. New Phytol. 2007: 176(4):782-91.

60. Zagari N, Sandoval-Ibañez O, Sandal N, Su J, Rodriguez-Concepcion M, Stougaard J, Pribil M, Leister D, Pulido P. SNOWY COTYLEDON 2 promotes chloroplast development and has a role in leaf variegation in both Lotus japonicus and Arabidopsis thaliana. Mol Plant. 2017;10(5):721-34.

61. D'Amici GM, Huber CG, Zolla L. Separation of thylakoid membrane proteins by sucrose gradient ultracentrifugation or blue native-SDS-PAGE twodimensional electrophoresis. In: Peirce MJ, Wait R, editors. Membrane proteomics: methods and protocols. Totowa: Humana Press; 2009. p. 60-70.

62. Zhao Y, Zhao C, Lu X, Zhou H, Li Y, Zhou J, Chang Y, Zhang J, Jin L, Lin F, et al. Investigation of the relationship between the metabolic profile of tobacco leaves in different planting regions and climate factors using a pseudotargeted method based on gas chromatography/mass spectrometry. J Proteome Res. 2013;12(11):5072-83.

63. Bolger AM, Lohse M, Usadel B. Trimmomatic: a flexible trimmer for Illumina sequence data. Bioinformatics. 2014;30(15):2114-20.

\section{Publisher's Note}

Springer Nature remains neutral with regard to jurisdictional claims in published maps and institutional affiliations.
Ready to submit your research? Choose BMC and benefit from:

- fast, convenient online submission

- thorough peer review by experienced researchers in your field

- rapid publication on acceptance

- support for research data, including large and complex data types

- gold Open Access which fosters wider collaboration and increased citations

- maximum visibility for your research: over $100 \mathrm{M}$ website views per year

At BMC, research is always in progress.

Learn more biomedcentral.com/submissions 